\title{
Nutrient-Gene Interactions
}

\section{A Genetic Polymorphism in SEPP1 Modifies Biomarkers of Selenium Status after Intake of Selenium-Rich Foods (E14-01)}

Gitte Ravn-Haren, ${ }^{1}$ Tine Iskov Kopp, ${ }_{1}$ Malene Outzen, ${ }^{2}$ Anja Olsen, ${ }^{2}$ and Ulla Vogel ${ }^{3}$

${ }^{1}$ National Food Institute, Technical University of Denmark; ${ }^{2}$ Danish Cancer Society Research Center, Denmark; and ${ }^{3}$ National Research Centre for the Working Environment, Denmark

Objective: The aim of this study was to investigate whether 4 functional single nucleotide polymorphisms (SNPs) in glutathione peroxidases (GPX1 and GPX4) and selenoprotein P (SEPP) modify the effect of intervention with selenium-rich foods on concentrations of blood selenium and selenoprotein expression (erythrocyte GPX activity and plasma SEPP concentration).

Methods: In a parallel dietary intervention study 83 healthy men and women aged 50-74 y were randomly assigned to either a control or an intervention group. Participants in the intervention group were provided with 5 portions of $200 \mathrm{~g}$ raw fish and shellfish once a week for $26 \mathrm{wk}$, corresponding to $\sim 50.3 \mu \mathrm{g} \mathrm{Se} / \mathrm{d}$. The participants in the control group received no intervention and were advised to maintain their habitual diets. Selected functional polymorphisms were GPX1/rs1050450, GPX4/rs713041, SEPP1/rs3877899, and SEPP1/rs7579. Genotypes were determined through the use of reverse transcriptase-polymerase chain reaction and allelic discrimination on ABI 7900HT instruments. Samples were run in duplicates with known positive controls and 3 negative controls. Duplicates yielded 100\% identical genotypes. Whole-blood selenium analyses were conducted by inductively coupled plasma mass spectrometry with an ELAN 6100 DRC, and plasma SEPP concentration was determined from its selective retention by heparin-affinity HPLC and online detection by inductively coupled plasma dynamic reaction cell mass spectrometry of selenium. GPX activity was spectrophotometrically assayed in erythrocyte lysates on a Pentra 400 with $t$-butylhydroperoxide as substrate and related to the amount of hemoglobin in the lysates.

Results: The intervention diet resulted in higher concentrations of both SEPP $(P=0.018)$ and blood selenium $(P=0.088)$ in wild-type allele carriers of the $S E P P 1 / \mathrm{rs} 3877899$ polymorphism compared with variant allele carriers. None of the other polymorphisms modified the biomarker responses following the intervention. Carriers of the variant GPX1 allele had significantly lower erythrocyte GPX enzyme activity compared with wild-type carriers independent of intervention.

Conclusions: Our study shows that variation in the SEPP1 gene modifies biomarkers of selenium status after intake of selenium-rich foods. This opens the way for a more personalized approach to micronutrient requirements.

\section{Funding Sources}

None.

Interactions between Diets High in Cholesterol and Dioxin-Like Pollutants Increase Inflammation, Disrupt Gut Microbiota, and Modulate Host Metabolism (E14-02)

Jessie Hoffman, Michael Petriello, Olga Vsevolozhskaya, Richard Charnigo, Pan Deng, Andrew Morris, and Bernhard Hennig

University of Kentucky

Objective: The gut microbiome is sensitive to diet and environmental exposures and is involved in the regulation of many host metabolic responses. Exposures to dioxin-like pollutants occur primarily via ingestion of contaminated foods and are linked to increased risk of developing metabolic diseases. Our objective was to elucidate the effects of the dioxin-like

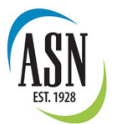

○

C) 2018 American Society for Nutrition. All rights reserved.

Manuscript received September 20, 2017. Initial review completed March 23, 2018. Revision accepted May 27, 2018. Published online June 28, 2018 
pollutant PCB 126 on gut health, gut microbiota, and metabolism in a well-established mouse model of chronic inflammatory diseases.

Methods: LDLr -/- mice were fed a high-cholesterol diet and exposed to PCB 126 or vehicle at weeks 2 and 4 of a 14-wk study. We utilized 16S rRNA sequencing, metabolomics, and inflammatory gene expression and protein quantification to examine the impact of PCB 126 on inflammation, the gut microbiome, and host metabolism.

Results: 16S rRNA sequencing showed that gut microbiota populations shifted at the phylum and genus levels in ways that mimic observations seen in metabolic inflammatory diseases. PCB 126 exacerbated the observed reduction in gut flora diversity and significantly increased the Firmicutes-to-Bacteroidetes ratio. PCB 126 exposure significantly increased circulating insulin levels without modulating glucose levels. Toxicant-exposed mice had a robust increase in hepatic long-chain fatty acids and dysregulated expression of enzymes related to lipid and carbohydrate metabolism. A significant correlation between glucagonlike peptide-1 (GLP-1) and Bifidobacterium was evident and dependent on toxicant exposure (statistical interaction was evident).

Conclusions: PCB 126 exposure disrupted the gut microbiota and host metabolism and increased intestinal and systemic inflammation. These data imply that the interaction between diet and dioxinlike pollutants may predispose an individual to the development of metabolic inflammatory diseases.

Funding Sources

P42ES007380.

Polymorphisms in IRS1 and FABP1 Modify Insulin Resistance in Response to Fatty Acid Composition in Nondiabetic Subjects with Abdominal Obesity (E14-03)

Shatha Hammad, ${ }^{1}$ Jyoti Sihag, ${ }^{1}$ Xiang Chen, ${ }^{1}$ Peter Eck, ${ }^{2}$ Philip Connelly, ${ }^{3}$ Benoit Lamarche, ${ }^{4}$ Patrick Couture, ${ }^{4}$ Kate Bowen, ${ }^{5}$ Sheila West, ${ }^{5}$ Penny Kris-Etherton, ${ }^{5}$ Carla Taylor, ${ }^{2}$ Peter Zahradka, ${ }^{2}$ David Jenkins, ${ }^{3}$ and Peter Jones ${ }^{1}$

${ }^{1}$ University of Manitoba, Richardson Centre for Functional Foods \& Nutraceuticals, Canada; ${ }^{2}$ University of Manitoba, Canada; ${ }^{3}$ University of Toronto, Canada; ${ }^{4}$ Laval University, Canada; and ${ }^{5}$ Pennsylvania State University

Background: Insulin resistance (IR) increases the risk of type 2 diabetes and several metabolic abnormalities. Emerging evidence suggests a modulatory effect of single-nucleotide polymorphisms (SNPs) on IR response to dietary fatty acid composition; yet, supportive evidence from clinical trials is missing.

Objective: We evaluated the impact of IR-associated genetic polymorphisms on the response of IR measures to different levels of dietary fatty acid composition.

Methods: Nondiabetic adults $(n=101)$ with abdominal obesity were included in a randomized, controlled-feeding, double-blind, crossover, multicenter trial. During each phase, participants consumed 1 of 3 treatment oils ( $20 \%$ of total fat) for $6 \mathrm{wk}$, separated by a 4 - to 12-wk washout. Treatment oils included two high-monounsaturated fatty acid (MUFA) oils, conventional canola [17.5\% energy MUFA, 9.2\% polyunsaturated fatty acid (PUFA), and 6.6\% saturated fatty acid (SFA)] or high-oleic acid canola (19.1\% energy MUFA, 7.0\% PUFA, 6.4\% SFA), and a low-MUFA high-SFA oil blend (11\% energy MUFA, 10\% PUFA,
$12 \%$ SFA). Genotyping of 9 candidate SNPs was performed through the use of a qualitative polymerase chain reaction system.

Results: Reductions in endpoint measures of insulin $(P=0.04)$, homeostasis model assessment (HOMA)- $\beta$ ( $\mathrm{P}=0.02)$ and HOMAIR $(P$-trend $=0.07)$ in the IRS1 rs7578326-GG homozygotes were observed upon consumption of the high-SFA diet compared with the high-MUFA diets. In the homozygotes of FABP1 rs2241883-CC, but not in other genotypes, reductions in HOMA-IR $(P=0.042)$ and fasting insulin ( $P$-trend $=0.06$ ) were also observed following consumption of the high-SFA diet compared with the high-MUFA diets. Polymorphisms within ADIPOQ, ADRB2, FTO, PLIN1, PPAR $\gamma$, and TCF7L2 genes failed to modulate the effect of fatty acid consumption on glucose homeostasis or IR measures.

Conclusion: Dietary fatty acid composition modified IR measures in the carriers of either the FABP1 rs2241883-CC or IRS1 rs7578326GG genotype, which might reflect a potential protective effect on insulin sensitivity following prolonged exposure to dietary high-SFA. These results may contribute to developing an effective genotypebased dietary recommendation to reduce IR incidence and associated complications.

This trial was registered at clinicaltrials.gov as NCT02029833.

\section{Funding Sources}

This research is part of the Canola Agri-Science Cluster, with funding provided by Agriculture and Agri-Food Canada, the Canola Council of Canada, Dow AgroSciences, Alberta Canola, SaskCanola and the Manitoba Canola Growers.

Tyrosol Bioconversion into Hydroxytyrosol in Humans: Regulation by CYP2A6 and CYP2D6 Polymorphisms (E14-04)

Anna Boronat Rigol, ${ }^{1}$ Julian Andrés Mateus, ${ }^{1}$ Natàlia Soldevila, ${ }^{2}$ Esther Menoyo, ${ }_{1}$ Clara Pérez-Mañá, ${ }^{2}$ Jose Rodríguez-Morató, ${ }^{2}$ Montserrat Fitó, ${ }^{1}$ Oliver Chen, ${ }^{3}$ Rachel F Tyndale, ${ }^{4}$ and Rafael de la Torre ${ }^{1}$

${ }^{1}$ IMIM-Hospital del Mar Medical Research Institute, Spain; ${ }^{2}$ Universitat Pompeu Fabra, Spain; ${ }^{3}$ Tufts University, MA; and ${ }^{4}$ University of Toronto, Canada

Objective: Tyrosol (TYR) is a phenol present in wine and olive oil. In humans, it is metabolized into hydroxytyrosol (HT), a very active antioxidant from olive oil and a metabolite of dopamine. Two isoforms of cytochrome P450, CYP2A6 and CYP2D6, regulate its formation. These isoenzymes are highly polymorphic, leading to genotypes with altered functionality. The objective of this study is to assess in humans the impact of CYP2A6 and CYP2D6 polymorphisms in the metabolic biotransformation of TYR into HT.

Methods: Thirty volunteers of both genders (mean \pm SD age: $65 \pm 5.6$ y) were given a white wine supplemented with TYR $(260 \mathrm{ml}$ of white wine and $50 \mathrm{mg} / \mathrm{d}$ for men; $130 \mathrm{ml}$ of white wine and $25 \mathrm{mg} / \mathrm{d}$ for women) for $4 \mathrm{wk}$. Urine samples were collected for $24 \mathrm{~h}$, over the last day of the intervention. The concentration of TYR and HT metabolites was determined through the use of LC-MS/MS to estimate the total recovery in urine. Furthermore, all volunteers were genotyped for CYP2A6 allelic variants ${ }^{*} 1,{ }^{*} 2,{ }^{*} 4,{ }^{*} 9,{ }^{*} 12,1 \times N$, and for CYP2D6 allelic variants ${ }^{*} 1,{ }^{*} 2,{ }^{*} 4,{ }^{*},{ }^{*} 10,{ }^{*} 35,{ }^{*} 41,1 \times N$, and $2 \times N$ through the use of TaqMan assays. Depending on their genotypes for both CYP2D6 and 
CYP2A6, individuals were categorized phenotypically as poor (PM), extensive (EM), or ultrarapid (UR) metabolizers. Individuals were considered globally PM if they presented at least one nonfunctional CYP. Recoveries of TYR and HT metabolites were compared following these phenotypes.

Results: The intake of wine supplemented with TYR significantly increased the recovery of TYR and HT metabolites compared with baseline. Individuals were categorized into 3 groups depending on their predicted global phenotype: PM $(n=9), \operatorname{EM}(n=17)$, and UR $(n=4)$. The recovery of TYR in the PM was statistically higher compared with EM. On the other hand, PM showed significantly lower concentrations of HT.

Conclusions: Polymorphisms in CYP2A6 and CYP2D6 are involved in the conversion of TYR into HT in humans. PM subjects are less efficient in biotransforming TYR into HT, and are therefore probably less susceptible to the health benefits associated with HT exposure.

\section{Funding Sources}

Funded by grants from Fondo de Investigación Sanitaria-ISCIIIFEDER (FIS PI14/00072 and predoctoral fellowship PFIS-FI16/00106 for ABR) and CIBEROBN (CB06/03/0028).

\section{Role of Hypoxia-Inducible Factor $1 \alpha$ in Oncostatin-Mediated Changes in Adipocyte Function (E14-05)}

\section{Dana M DeSilva and Ron Morrison}

University of North Carolina at Greensboro

Objective: Obesity leads to chronic inflammation and hypoxia in adipose tissue. Research has shown that the inflammatory cytokine oncostatin M (OSM) induces the transcription factor hypoxia-inducible factor $1 \alpha(\mathrm{HIF}-1 \alpha)$ in a cell-type-specific manner. The objective of this study was to investigate the regulation and mechanism of OSM-induced HIF- $1 \alpha$ in adipocytes and the impact on adipocyte bioenergetics and adipokine secretion. We hypothesized that OSM causes a shift toward anaerobic metabolism and regulates adipokine secretion through mechanisms involving HIF- $1 \alpha$.

Methods: We stimulated 3T3-L1 adipocytes with hypoxia mimetic $\mathrm{CoCl}_{2}$ and cytokine OSM, collected RNA and protein, and used reverse transcriptase-polymerase chain reaction (RT-PCR) and western blotting to explore the regulation of HIF- $1 \alpha$ under these conditions. Additionally, to explore mechanisms by which OSM-induced transcription of Hif- $1 \alpha$, we investigated known OSM signaling cascades that use specific pathway inhibitors. RT-PCR was also used to determine OSM-induced changes in gene expression that impact glucose uptake, glycolytic metabolism, and adipokine secretion. A regulatory role for HIF- $1 \alpha$ in OSM-mediated changes in adipocyte function was examined by siRNA.

Results: Our data reveal that hypoxia and inflammation have different mechanisms of HIF- $1 \alpha$ upregulation. Whereas hypoxia mimetics stabilize HIF- $1 \alpha$ protein, OSM regulates Hif- $1 \alpha$ transcriptionally. We also found that the transcriptional regulation of OSM-mediated HIF- $1 \alpha$ is dependent on ERK, AKT, and STAT signaling pathways. The combination of both $\mathrm{CoCl}_{2}$ and OSM stimulation led to a synergistic effect on HIF- $1 \alpha$ protein accumulation and target gene regulation. Data also revealed a direct role for HIF- $1 \alpha$ in mediating OSM-induced changes in adipocyte bioenergetics and adipokine secretion.
Conclusions: Our data demonstrate that stimulation of 3T3-L1 with $\mathrm{CoCl}_{2}$ and OSM induces HIF- $1 \alpha$ and causes changes in adipocyte function. Although it is known that hypoxia and inflammation underlie changes that lead to obesity-associated disorders, these roles in adipocytes have yet to be fully elucidated. These data are significant as understanding the mechanisms and effects of hypoxia and inflammation are critical to understanding adipocyte dysfunction.

\section{Funding Sources}

NIH-NIDDK (R15-DK082799).

Maternal Fatty Acid Desaturase Single-Nucleotide Polymorphisms, Prenatal Supplementation with Docosahexaenoic Acid, and Child Size at 5 Years of Age (E14-06)

Meriah Schoen, ${ }^{1}$ Ines Gonzalez-Casanova, ${ }^{2}$ Peter Rzehak, ${ }^{3}$ Eva Reischl, ${ }^{4}$ Aryeh Stein, ${ }^{2}$ Albino Barraza-Villarreal, ${ }^{5}$ Raquel Garcia Feregrino, ${ }^{5}$ Juan Rivera Dommarco, ${ }^{5}$ Isabelle Romieu, ${ }^{6}$ Salvador Villalpando, ${ }^{5}$ Berthold Koletzko, ${ }^{3}$ and Usha Ramakrishnan ${ }^{2}$

${ }^{1}$ Emory University, GA; ${ }^{2}$ Rollins School of Public Health, Emory University, GA; ${ }^{3}$ Ludwig-Maximilians-University of Munich, Germany; ${ }^{4}$ Helmholtz Zentrum München, Germany; ${ }^{5}$ National Institute of Public Health, Cuernavaca, Mexico; and ${ }^{6}$ World Health Organization

Objectives: Maternal fatty acid desaturase (FADS) single-nucleotide polymorphisms (SNPs) modify the impact of prenatal docosahexaenoic acid (DHA) supplementation on offspring birth weight. Prior work has shown that the offspring of carriers of the TT and TC alleles for the SNP rs174602 who received prenatal DHA supplements were heavier at birth than controls. This analysis examined whether maternal FADS SNPs continue to modify the impact of this intervention on offspring length and body mass index (BMI) at $5 \mathrm{y}$ of age.

Methods: We analyzed data from POSGRAD, a randomized controlled trial of prenatal DHA supplementation conducted in Mexico (NCT00646360). Pregnant women were randomly assigned to receive $400 \mathrm{mg} / \mathrm{d}$ of preformed DHA or placebo from 18 to $22 \mathrm{wk}$ of gestation through delivery. Their children have been followed prospectively. Maternal genotype was derived from stored blood samples, and 5 target SNPs (rs174455, rs174556, rs174602, rs498793, rs2727271) that represent the FADS1, FADS2, and FADS3 genes were analyzed. We estimated differences in offspring height-for-age $z$ score (HAZ), BMIfor-age $z$ score (BAZ), and stunting (HAZ $<-2)$ at $5 \mathrm{y}$ of age between DHA $(n=352)$ and placebo $(\mathrm{n}=340)$, stratified by FADS SNPs.

Results: Maternal characteristics were similar for the DHA and control groups. Mean maternal age, height, and BMI $\left(\mathrm{kg} / \mathrm{m}^{2}\right)$ at recruitment were $26.5 \pm 4.8 \mathrm{y}, 155.1 \pm 5.7 \mathrm{~cm}$, and $26.3 \pm 4.4$. Mean minor allele frequencies were $6.5 \%$ for rs $174455,5.2 \%$ for $\mathrm{rs} 174556$, $14.9 \%$ for rs $174602,19.3 \%$ for rs 498793 , and $21.8 \%$ for rs 2727271 . Mean offspring HAZ and BAZ at $5 \mathrm{y}$ of age were $-0.40 \pm 0.89$ and $0.13 \pm 1.11$, respectively. Stunting prevalence was $3.6 \%$, and the prevalence of BAZ $>1$ and $\mathrm{BAZ}<-1$ were $19.2 \%$ and $12.1 \%$, respectively. Maternal height and BMI were significantly associated with offspring HAZ, but there was no significant heterogeneity across the 5 target SNPs and prenatal DHA for all anthropometric measures at $5 \mathrm{y}$ of age ( $P>0.05$ for all analyses).

Conclusions: Maternal FADS genotypes did not modify the null effect of prenatal DHA supplementation on child length and BMI at $5 \mathrm{y}$ of age. It is possible that maternal genotype plays a greater role on DHA 
availability during fetal growth, whereas offspring genotype and dietary intakes may be more important later in childhood.

Funding Sources

NIH, CONACYT, the March of Dimes and Nutricia Foundation.

Genetic Variants of Gestational Diabetes Mellitus: A Study of 112 Single-Nucleotide Polymorphisms among 8722 Women in Two Independent Populations (OR15-01)

Ming Ding, ${ }^{1}$ Jorge Chavarro, ${ }^{2}$ Sjurdur Olsen, ${ }^{3}$ Yuan Lin, ${ }^{4}$ Sylvia Ley, ${ }^{2}$ Wei Bao, ${ }^{5}$ Shristi Rawal, ${ }^{6}$ Louise Grunnet, ${ }^{7}$ Anne Thuesen, ${ }^{7}$ James Mills, ${ }^{4}$ Edwina Yeung, ${ }^{4}$ Stefanie Hinkle, ${ }^{4}$ Wei Zhang, ${ }^{3}$ Allan Vaag, ${ }^{8}$ Aiyi Liu, ${ }^{4}$ Frank Hu, ${ }^{2}$ and Cuilin Zhang ${ }^{4}$

${ }^{1}$ Harvard TH Chan School of Public Health, MA; ${ }^{2}$ Harvard School of Public Health, MA; ${ }^{3}$ Statens Serum Institut, Copenhagen, Denmark; ${ }^{4}$ National Institutes of Health; ${ }^{5}$ University of Iowa; ${ }^{6}$ Rutgers University, NJ; ${ }^{7}$ Rigshospitalet University Hospital, Copenhagen, Denmark; and ${ }^{8}$ Early Clinical Development and Innovative Medicines, Mölndal, Sweden

Background: Gestational diabetes mellitus (GDM) is a common pregnancy complication that has substantial short- and longterm adverse health implications for women and their children. However, large-scale studies on genetic risk loci for GDM remain sparse.

Methods: We conducted a case-control study among 2636 GDM cases and 6086 non-GDM controls from the Nurses' Health Study II (NHSII) and the Danish National Birth Cohort (DNBC). One hundred and twelve susceptibility genetic variants confirmed by genome-wide association studies for type 2 diabetes (T2D) were selected and measured. A weighted genetic risk score (GRS) was created based on variants that were significantly associated with risk of GDM after correcting for false discovery rate (FDR).

Results: For the first time, we identified 8 variants associated with GDM, namely rs7957197 (HNF1A), rs10814916 (GLIS3), rs3802177 (SLC30A8), rs9379084 (RREB1), rs34872471 (TCF7L2), rs7903146 (TCF7L2), rs11787792 (GPSM1), and rs7041847 (GLIS3). Additionally, we confirmed 3 variants, rs10830963 (MTNR1B), rs1387153 (MTNR1B), and rs4506565 (TCF7L2), that were previously significantly associated with GDM risk. Furthermore, compared with participants in the first (lowest) quartile of weighted GRS based on these 11 singlenucleotide polymorphisms (SNPs), the ORs of GDM were 1.07 (95\% CI: $0.93,1.22), 1.23$ (95\% CI: 1.07, 1.41), and 1.53 (95\% CI: 1.34, 1.74) in the second, third, and fourth (highest) quartiles, respectively. The significant positive associations between the weighted GRS and risk of GDM persisted across most of the strata of major risk factors for GDM, including family history of type 2 diabetes, smoking status, body mass index, and age.

Conclusion: In this large-scale case-control study with women from 2 independent populations, 8 novel GDM SNPs were identified, which offers potential to improve our understanding of GDM etiology.

\section{Funding Sources}

This work was supported by the Intramural Research Program of the Eunice Kennedy Shriver National Institute of Child Health and Human Development at the National Institutes of Health (contracts HHSN275201000020C, HHSN275201500003C, HHSN275201300026I, HSN275201100002I). The Nurses' Health Study II cohort is supported by the National Institutes of Health (grant UM1 CA176726, R01 CA50385, and NICHD contract HHSN275201000020C). Financial support for the Danish component was received from: March of Dimes Birth Defects Foundation (6-FY-96-0240, 6-FY97-0553, 6-FY970521, 6-FY00-407), Innovation Fund Denmark (grants 09-067124 and 11-115923, "Centre for Fetal Programming"), the Health Foundation (11/263-96), the Heart Foundation (96-2-4-83-22450), and the EU (FP7-289346-EarlyNutrition).

Leptin Gene Polymorphism (G2548A) Is Associated with Serum Lipid Changes and Dietary Intake during Pregnancy: A Prospective Cohort Study (OR15-02)

Dayana Rodrigues Farias, ${ }^{1}$ Nadya Alves-Santos, ${ }^{1}$ Ilana Eshriqui, ${ }^{2}$ Maisa Martins, ${ }^{1}$ Claudio J Struchiner, ${ }^{3}$ Jaqueline Lepsch, ${ }^{4}$ Nathalia Costa, ${ }^{1}$ and Gilberto $\mathrm{Kac}^{1}$

${ }^{1}$ Federal University of Rio de Janeiro, Brazil; ${ }^{2}$ University of São Paulo, Brazil; ${ }^{3}$ Oswaldo Cruz Foundation, Brazil; and ${ }^{4}$ Santa Ursula University, Brazil

Objectives: The aims of this study were to evaluate the associations between leptin and leptin receptor gene single-nucleotide polymorphisms (SNPs) and serum lipid concentrations during pregnancy, and to determine whether dietary intake is a mediator in these associations.

Methods: A prospective cohort was followed in Rio de Janeiro, Brazil, during pregnancy (weeks 5-13, 20-26, and 30-36). High-density lipoprotein (HDL), total cholesterol (TC), and triglycerides (TGs) were measured by enzymatic colorimetric method, and low-density lipoprotein (LDL) cholesterol was calculated. DNA was extracted by the phenol-chloroform method and leptin (G2548A, rs7799039) and leptin receptor SNPs (Q223R; rs1137101 and K109R; rs1137100) were genotyped through the use of real-time polymerase chain reaction. Dietary intake was assessed at the third trimester through the use of a food-frequency questionnaire. Statistical analyses included linear mixed-effect models adjusted for skin color, prepregnancy body mass index (BMI) and total fat dietary intake.

Results: A total of 154 women were evaluated in the first, 147 in the second, and 149 in the third pregnancy trimester. Women were (mean $\pm \mathrm{SD}$ ) aged $26.8 \pm 5.5 \mathrm{y}$, had $8.6 \pm 3.0 \mathrm{y}$ of schooling, and a BMI (in $\mathrm{kg} / \mathrm{m}^{2}$ ) of $24.9 \pm 4.7$. A total of 59 (38.3\%) women were nulliparous and 117 (75.5\%) reported mixed or black skin color. Women with the AA genotype of G2548A SNP had a higher energy percentage of total fat $(29.1 \pm 4.4 \%$ compared with $25.0 \pm 4.3 \% \mathrm{kcal} ; P<0.001)$ and total energy intake $(2878 \pm 1405$ compared with $2354 \pm 708$ kcal; $P=0.016)$ and presented a higher increase per gestational week (interaction SNP $\times$ time) in serum TC $(\beta=0.7 ; 95 \% \mathrm{CI}:-0.04,1.4$; $P=0.066)$ and TG $(\beta=1.2 ; 95 \% \mathrm{CI}: 0.2,2.2 ; P=0.022)$ than those with AG/GG genotypes. The association between G2548A SNP and serum lipids was not attenuated when analysis was adjusted for dietary intake (Supporting figure). Only nonsignificant associations were observed between Q223R and K109R SNPs and serum lipids or dietary intake, or between G2548A SNP and cholesterol fractions.

Conclusions: Women homozygotous for the A-allele of the leptin SNP (G2548A) presented higher total energy and fat intake during pregnancy and higher increase in TC and TG concentrations per gestational week, compared with AG or GG genotypes. Leptin receptor SNPs were not associated with serum lipids or dietary intake. 
Supporting Images/Graphs
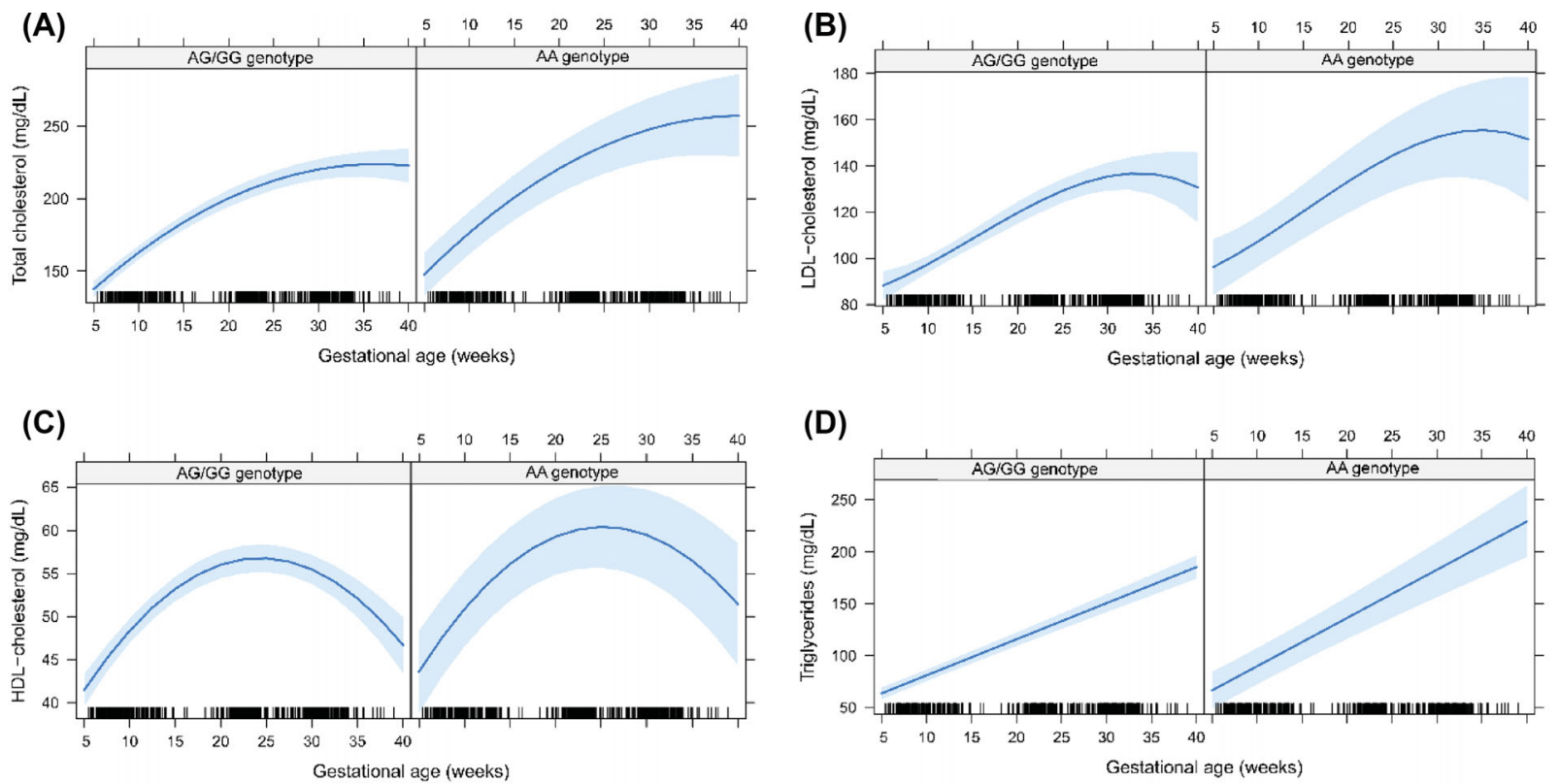

FIGURE OR15-02-1 Longitudinal change in total cholesterol (A), LDL-cholesterol (B), HDL-cholesterol (C) and Triglycerides (D) during pregnancy according to leptin gene polymorphism genotypes (G2548A) in 154 women followed at a public health center in Rio de Janeiro, Brazil, $2009-2012$.

Note: p-valuc refers to maximun likelihood estimator. $\beta=$ linear mixed-effect cocfficient ( $95 \%$ confidence interval). The models were adjusted for dietary intake, gestational week (and quadratic gestational wee (CT, HDI and IDL) and cubic gestational week (LDI), the interaction hetween leptin gene polymorphism and gestational week (time), pre-pregnancy BMI and skin color, a) $\beta$.

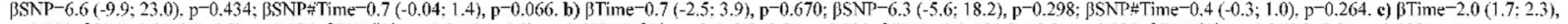

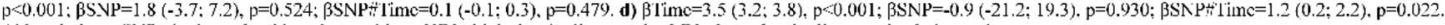

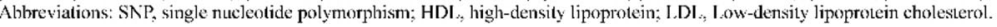

\section{Funding Sources}

The study was funded by the National Council for Scientific and Technological Development (CNPq) and the Carlos Chagas Filho Foundation for Research Support of Rio de Janeiro State (FAPERJ).

Maternal Fatty Acid Desaturase Single-Nucleotide Polymorphism Modifies the Impact of Prenatal Docosahexaenoic Acid Supplementation on Offspring Development at 5 Years of Age (OR15-03)

Ines Gonzalez-Casanova, ${ }^{1}$ Meriah Shoen, ${ }^{1}$ Peter Rzehak, ${ }^{2}$ Aryeh Stein, ${ }^{1}$ Albino Barraza-Villarreal, ${ }^{3}$ Eva Reischl, ${ }^{4}$ Raquel Garcia Feregrino, ${ }^{3}$ Ann DiGirolamo, ${ }^{5}$ Lourdes Schnaas, ${ }^{6}$ Juan Rivera, ${ }^{3}$ Isabelle Romieu, ${ }^{7}$ Salvador Villalpando, ${ }^{3}$ Berthold Koletzko, ${ }^{2}$ and Usha Ramakrishnan ${ }^{2}$

${ }^{1}$ Emory University, GA; ${ }^{2}$ Ludwig-Maximilians-University of Munich, Germany; ${ }^{3}$ Mexican National Institute of Public Health; ${ }^{4}$ Helmholtz Zentrum München, Germany; ${ }^{5}$ Georgia Health Policy Center, Georgia State University; ${ }^{6}$ National Institute of Perinatology; and ${ }^{7}$ International Agency for Cancer Research

Objectives: Maternal single-nucleotide polymorphisms (SNPs) in the fatty acid desaturase genes (FADS) modulate the conversion of dietary long-chain polyunsaturated fatty acids into docosahexaenoic acid (DHA), which accretes in the brain during early development. We examined whether maternal FADS SNPs modified the impact of prenatal DHA supplementation on measurements of offspring cognitive development at $5 \mathrm{y}$ of age.

Methods: As part of a randomized clinical trial in Mexico, 1094 women received $400 \mathrm{mg} / \mathrm{d}$ of preformed DHA or a placebo from gestation week 18-22 through delivery. For this analysis, we included the offspring who completed the McCarthy Scales of Children's Abilities (MSCA) at $5 \mathrm{y}$ and with maternal FADS genotype information $(\mathrm{DHA}=350$; control $=338$ ). We included five target FADS SNPs (rs174455, rs174556, rs174602, rs498793, rs2727271) and used MANOVA to test for interactions between them and prenatal DHA with verbal, perception, quantitative, and memory raw scores as outcomes. We adjusted all models for child age at measurement.

Results: The DHA and control groups were similar with respect to many maternal and offspring characteristics. At baseline, mean $\pm \mathrm{SD}$ maternal age was $26 \pm 5 \mathrm{y}$ and years of schooling was $12 \pm 4 ; 46 \%$ of the children were female and mean birth weight was $3215 \pm 469$ g. Raw scores for the MSCA scales were: verbal $53 \pm 12$; perception $47 \pm 9$; quantitative $20 \pm 6$; and memory $25 \pm 8$. There were no intent-to-treat differences between DHA and control groups. We found a significant interaction by SNP $\operatorname{rs} 174602(P<0.01)$; among offspring of TT (minor allele homozygotes), those in the DHA group had higher scores in the quantitative (DHA $=23 \pm 7$ compared with control $=19 \pm 6 ; P=0.01$ ) and memory (DHA $=28 \pm 8$ compared with control $=24 \pm 8 ; P=0.01$ ) scales, whereas there were no significant differences between DHA and control among TC or CC carriers. We found no significant heterogeneity due to the other 4 FADS SNPs.

Conclusions: A maternal SNP in the FADS2 coding region modified the impact of prenatal DHA on cognitive development outcomes at $5 \mathrm{y}$ of age. Differential responses to prenatal DHA supplementation based on target population genetic make-up could contribute to elucidate results from previous supplementation trials. 
Funding Sources

Funded by NIH, CONACYT, the March of Dimes and Nutricia Foundation.

TCF7L2 Gene and Mediterranean Diet Interactions Modulating Anthropometric Factors in Puerto Rican Adults (OR15-04)

Mercedes Sotos Prieto, ${ }^{1,2}$ Caren Smith, ${ }^{3}$ Chao-Qiang Lai, ${ }^{3,4}$ Jose Maria Ordovas, ${ }^{3}$ Katherine L Tucker, ${ }^{5}$ and Josiemer Mattei ${ }^{2}$

${ }^{1}$ Ohio University; ${ }^{2}$ Harvard TH Chan School of Public Health, MA; ${ }^{3}$ Jean Mayer-USDA Human Nutrition Research Center on Aging at Tufts University, MA; ${ }^{4}$ Agricultural Research Service, USDA; and ${ }^{5}$ University of Massachusetts Lowell

Objectives: Polymorphisms in the TCF7L2 gene that predispose to type 2 diabetes (T2D) show inconsistent associations with anthropometric factors. Interaction with dietary factors may explain these observations. We aimed to examine the potential modulation of TCF7L2 rs7903146 and rs12255372 on anthropometric factors by the Mediterranean diet (MD) among Puerto Ricans living in Boston, who have a high prevalence of T2D.

Methods: In this cross-sectional analysis, we genotyped TCF7L2rs7903146 (TT: 9\%; CT: 43\%; CC: 48\%) and rs12255372 (TT: 8\%; GT: 42\%; GG: 50\%) in 1381 participants (aged 45-75y) of the Boston Puerto Rican Health Study. Anthropometric factors were measured and diet was collected by validated questionnaire. An MD score was defined based on adherence to 9 food and nutrient components based on sexspecific population-based median cutoffs (high $\mathrm{MD}=$ meeting $>4$ components).

Results: MD score interacted significantly with rs7903146, with risk $\mathrm{T}$ allele carriers having lower means of factors at high MD adherence than CC individuals, on weight $(\mathrm{CT}+\mathrm{TT}: 77.3 \pm 0.99 \mathrm{~kg}$ compared with CC: $80.9 \pm 1.03 \mathrm{~kg} ; P$-interaction $=0.026)$ and waist circumference (WC) (CT + TT: $99.2 \pm 0.89 \mathrm{~cm}$ compared with CC: $102.2 \pm 99.2 \mathrm{~cm}$; $P$-interaction $=0.026)$. The interaction was marginally significant for body mass index (BMI; $P=0.06$ ). For rs12255372, compared with GG individuals, those with the risk $T$ allele showed lower weight $\left(\mathrm{kg} / \mathrm{m}^{2} \mathrm{GG}\right.$ : $80.3 \pm 1.03$; GT: $78.4 \pm 1.08$; TT: $75.7 \pm 2.58 \mathrm{~kg}$; $P$-interaction $=0.037$ ) and BMI $\left(\mathrm{kg} / \mathrm{m}^{2}\right.$; GG: $32.0 \pm 0.39$; GT: $31.4 \pm 0.41$; TT: $30.6 \pm 0.99$, $P$-interaction $=0.031)$ at high MD adherence. No significant interaction was found for WC, although the rs12255372 TT genotype showed lower WC at high MD adherence $(97.6 \pm 2.29 \mathrm{~cm})$ compared with low MD adherence $(104.6 \pm 2.13 \mathrm{~cm})$. At low MD adherence, anthropometric factors were similar by rs7903146 genotype and higher for T carriers of rs12255372.

Conclusions: Individuals with the TCF7L2 rs7903146 and rs12255372 T2D risk genotypes had better anthropometric profiles when adhering to an $\mathrm{MD}$, suggesting that unfavorable predisposition may be curbed by a healthy diet. Studies should clarify the role of diet and risk alleles on anthropometric factors on populations at risk of T2D to help with precision medicine.

\section{Funding Sources}

NIH-National Heart, Lung, and Blood Institute grants K01HL120951 and P50-HL105185; National Institute on Aging grant P01AG023394. NIH P50HL105185, P01AG023394, R01AG055948.This work was partially funded by the US Department of Agriculture, agreement no. 8050-51000-098-00D.

\section{Biological Insights from Genetics of Breakfast Intake (OR15-05)}

Hassan S Dashti, ${ }^{1}$ Jordi Merino, ${ }^{1}$ Jacqueline M Lane, ${ }^{1}$ Traci M Bartz, ${ }^{2}$ Nicola M McKeown, ${ }^{3}$ Caren Smith, ${ }^{3}$ Toshiko Tanaka, ${ }^{4}$ Rozenn N Lemaitre, ${ }^{2}$ Martin K Rutter, ${ }^{5}$ and Richa Saxena ${ }^{1}$

${ }^{1}$ Massachusetts General Hospital; ${ }^{2}$ University of Washington; ${ }^{3}$ Tufts University, MA; ${ }^{4}$ National Institute on Aging; and ${ }^{5}$ University of Manchester, United Kingdom

Objectives: Breakfast consumption is a heritable dietary behavior associated with lower body weight and cardiometabolic disease risk, yet variation exists in whether breakfast is consumed. Discovery of genetic variants influencing diet beyond composition is hindered by the lack of data on meal preference captured by traditional dietary assessment methods. The objectives of this study were to identify genetic factors that influence breakfast intake and unravel shared biological links with other traits.

Methods: We leveraged the power of the UK Biobank $(n=500,000)$ to identify genetic variants related to breakfast cereal intake as a proxy for breakfast. Breakfast cereal frequency was estimated based on data from up to 5 web-based 24-h diet recalls, and responses were categorized as "never," "sometimes," and "always" ( $n=193,860)$. We performed a genome-wide association study (GWAS) that used linear mixed models adjusting for age, sex, 10 principal components, and genotyping array in related participants of European ancestry. We then verified observed links in the Cardiovascular Health Study (CHS) that captured breakfast intake frequency as assessed by "How often do you eat breakfast?" ("never" compared with "always") by performing GWAS through the use of age- and sex-adjusted logistic regression $(n=3044)$.

Results: From the UK Biobank, we identified 6 independent genome-wide significant loci. Associations included genes implicated for caffeine intake/metabolism (CYP1A1), carbohydrate intake (FGF21), and schizophrenia (ZNF804A). Genome-wide genetic correlations indicated shared biological links between infrequent breakfast and insomnia, depression, obesity, coronary artery disease, and smoking $\left(P<2.14 \times 10^{-4}\right)$. We also observed that a genetic-risk score for higher coffee intake was associated with lower breakfast intake $(P<0.05)$, suggesting shared genetics among foods. In the CHS, we confirmed links with insomnia and schizophrenia through the use of genetic-risk scores for those traits $(P<0.05)$. Replication analyses to confirm UK Biobank top signals are underway upon inclusion of other CHARGE consortium cohorts.

Conclusions: The present findings provide a novel understanding of the genetic architecture of breakfast behavior including shared genetic links.

Highly Processed Food Intake Is Associated with Accelerated Epigenetic Aging (OR15-06)

Silvia Berciano, ${ }^{1}$ Jose Ordovas, ${ }^{1}$ and Chao-Qiang Lai ${ }^{2}$

${ }^{1}$ Jean Mayer USDA Human Nutrition Researce Center on Aging at Tufts University, MA; and ${ }^{2}$ USDA Agricultural Research Services 
Objective: DNA methylation is an essential epigenetic mechanism that regulates gene expression and downstream biological processes. Estimates of biological age based on DNA methylation patterns, often referred to as epigenetic age (DNAm age), strongly correlate with chronologic age in humans. The objective of this study was to determine whether the degree of divergence between epigenetic age and chronologic age is associated with habitual diet in a community-based cohort.

Methods: Methylation data sets from 2568 participants in exam 8 of the Framingham Heart Study Offspring Cohort were obtained from the NCBI dbGAP database of genotypes and phenotypes to be used in the current analyses. Genome-wide methylation was profiled with the use of the Illumina Infinium HumanMethylation450K BeadChip, and DNAm age was calculated with the use of the Horvath DNAm Age Calculator. Delta age was calculated as DNAm age minus chronologic age. Dietary assessment was conducted based on a validated 126item food-frequency questionnaire. Linear regression models were used to examine the relationship between delta age and dietary factors, adjusting for age, sex, and energy intake. $P$ values were then adjusted to account for multiple comparisons. Adjusted $P$ values $<0.05$ were considered statistically significant.

Results: DNAm age and chronologic age were highly correlated in the study sample $\left(r^{2}=0.78\right)$. Delta age was positively associated with intake of highly processed foods (adjusted $P$ value $<0.05$ ), suggesting a link between processed food intake and accelerated aging. Conversely, the consumption level of highpolyphenol foods was negatively associated with delta age (adjusted $P$ value $<0.05)$

Conclusions: Our findings suggest that habitual diet could influence aging rate via epigenetic mechanisms. Further research is required to evaluate the effectiveness of targeted dietary interventions for optimization of biological aging rate through modulation of methylation levels at specific age-related $\mathrm{CpG}$ sites.

\section{Funding Sources}

This work was funded by the US Department of Agriculture, agreement no. 8050-51000-098-00D.

Epigenomics and Metabolomics Reveal the Mechanism of the APOA2-Saturated Fat Intake Interaction Affecting Obesity (OR1507)

Chao-Qiang Lai, ${ }^{1}$ Caren Smith, ${ }^{2}$ Laurence D Parnell, ${ }^{1,2}$ YuChi Lee, ${ }^{2}$ Dollores Corella, ${ }^{3}$ Paul Hopkins, ${ }^{4}$ Bertha A Hidalgo, ${ }^{5}$ Stella Aslibekyan, ${ }^{5}$ Michael Province, ${ }^{6}$ Devin Absher, ${ }^{7}$ Donna K Arnett, ${ }^{8}$ Katherine L Tucker, ${ }^{7}$ and Jose M Ordovas ${ }^{2}$

${ }^{1}$ USDA Agricultural Research Services; ${ }^{2}$ Jean Mayer USDA Human Nutrition Research Center on Aging at Tufts University, MA; ${ }^{3}$ University of Valencia and CIBER Physiopathology of Obesity and Nutrition, Valencia, Spain; ${ }^{4}$ University of Utah; ${ }^{5}$ School of Public Health, University of Alabama; ${ }^{6}$ Washington University School of Medicine, MO; ${ }^{7}$ HudsonAlpha Institute for Biotechnology, AL; and ${ }^{8}$ College of Public Health, University of Kentucky

Objective: A putative functional variant $-265 \mathrm{~T}>\mathrm{C}$ (rs5082) within the $A P O A 2$ promoter has shown consistent interactions with saturated fat (SFA) intake to influence risk of obesity. This aim of this study was to take an integrative approach to characterize the molecular basis of this interaction.

Design: We conducted an epigenome-wide scan on 80 participants carrying either the rs5082 CC or TT genotypes and consuming either a low ( $<22 \mathrm{~g} / \mathrm{d})$ or high $(\geq 22 \mathrm{~g} / \mathrm{d})$ SFA diet, matched for age, sex, and diabetes in the Boston Puerto Rican Health Study (BPRHS). We then validated the findings in selected participants in the Genetics of Lipid Lowering Drugs and Diet Network (GOLDN) $(n=379)$ and the Framingham Heart Study (FHS) $(n=243)$. Transcription and metabolomics analyses were conducted to determine the relationship between epigenetic status, APOA2 mRNA expression, and blood metabolites.

Results: In BPRHS subjects, we identified methylation site cg04436964 as exhibiting significant differences between CC and TT participants consuming a high-SFA diet, but not among those consuming a low-SFA diet. Similar results were observed in GOLDN and FHS. Additionally, in FHS, cg04436964 methylation was negatively correlated with $A P O A 2$ expression in participants consuming a high-SFA diet. Furthermore, when consuming a high-SFA diet, CC carriers had lower APOA2 expression compared with those with the TT genotype. Finally, metabolomic analysis identified 4 pathways as overrepresented by metabolite differences between CC and TT genotypes with high SFA intake, including tryptophan and branchedchain amino acid (BCAA) pathways. Interestingly, these pathways were linked to rs5082-specific cg04436964 methylation differences in in participants consuming a high-SFA diet.

Conclusions: The epigenetic status of $A P O A 2$ is associated with SFA intake and $A P O A 2-265 \mathrm{~T}>\mathrm{C}$ genotype, promoting an APOA2 expression difference between APOA2 genotypes on a high-SFA diet, and modulating BCAA and tryptophan metabolic pathways. These findings identify potential mechanisms by which this highly reproducible gene-diet interaction influences obesity risk.

Funding Sources

This work was funded by the USDA, under agreement no. 805051000-098-00D, and by National Heart, Lung, and Blood Institute grant U01-HL072524-04 and R01HL104135, and NIH grant P01 AG023394, P50 HL105185, R01 AG027087. CES is supported by K08 HL112845.

An Integrative Analysis of Host, Gut Microbiota, and Environment Highlights the Link between Insulin Sensitivity and BranchedChain Amino Acids in Calorie Restriction (OR15-08)

Maria Carlota Dao, ${ }^{1}$ Nataliya Sokolovska, ${ }^{2}$ Rémi Brazeilles, ${ }^{3}$ Séverine Affeldt, ${ }^{4}$ Edi Prifti, ${ }^{4}$ Julien Chilloux, ${ }^{5}$ Eric Verger, ${ }^{4}$ Brandon Kayser, ${ }^{4}$ Judith Aron-Wisnewsky, ${ }^{4}$ Véronique Pelloux, ${ }^{6}$ Farid Ichou, ${ }^{4}$ Estelle Pujos-Guillot, ${ }^{7}$ Lesley Hoyles, ${ }^{5}$ Catherine Juste, ${ }^{8}$ Joel Doré, ${ }^{8}$ Marc-Emmanuel Dumas, ${ }^{5}$ Salwa W Rizkalla, ${ }^{4}$ Bridget Holmes, ${ }^{3}$ Jean-Daniel Zucker, ${ }^{4}$ and Karine Clément ${ }^{4}$

${ }^{1}$ Jean Mayer USDA Human Nutrition Research Center on Aging at Tufts University, MA; ${ }^{2}$ Sorbonne Universités, UPMC University Paris, France; ${ }^{3}$ Danone Nutricia Research, France; ${ }^{4}$ Institute of Cardiometabolism and Nutrition, ICAN, Assistance Publique Hôpitaux de Paris, Pitié-Salpêtrière Hospital, France; ${ }^{5}$ Imperial College London, Section of Biomolecular Medicine, Division of Computational and Systems Medicine, Department of Surgery and Cancer, Faculty of Medicine, United Kingdom; ${ }^{6}$ INSERM, France; ${ }^{7}$ Université Clermont 
Auvergne, INRA, Unité de Nutrition Humaine, France; and ${ }^{8}$ Micalis Institute, INRA, AgroParisTech, Université Paris-Saclay, France

Objectives: Lifestyle factors and gut microbiota play a role in the maintenance of insulin sensitivity (IS), yet the underlying mechanisms are only partially understood. Individual response to calorie restriction (CR) interventions in obese people may lead to different effects on IS. Through deep phenotyping and data integration approaches, greater insight could be achieved into the study of host, environment, and gut microbiota interactions. We took a data integration approach to investigate associations between change in IS and variations in lifestyle factors, host characteristics, and gut microbiota composition after CR in overweight and obese adults.

Methods: Overweight/obese adults $(n=27)$ undergoing a 6-wk CR period were extensively phenotyped at baseline and week 6 , including bioclinical parameters, subcutaneous adipose tissue (sAT) transcriptomics (Ilumina), metabolomics in serum, urine (nuclear magnetic resonance spectroscopy, Bruker) and feces (GC-MS, Agilent), food and nutrient intake (7-d food records), and gut microbiota metagenomics (SOLiD sequencing). Partial least-squares with canonical method was used to determine the associations of change (week 6 - baseline) between IS markers and groups of variables from host, microbiota, and environment. The Spectral Consensus Strategy (developed by us) was used for reconstruction of biological networks.

Results: A spectrum of variables from gut microbiota, lifestyle factors, and host with variations most strongly associated with improvements in IS was identified. Most notably, the biological networks highlighted relationships between variations in IS, serum branchedchain amino acids (BCAAs), sAT genes involved in endoplasmic reticulum (ER) stress and ubiquitination, metabolites, and gut metagenomic species.

Conclusions: Our approach of integrating high-dimensional data and selecting elements most strongly associated with CR-induced IS improvement led to confirmation of known links with IS (namely, BCAAs) and identification of new ones (sAT genes involved in ER stress and ubiquitination). Upon further validation in larger cohorts, this approach will lead to the identification of modifiable factors and biomarkers that may be used to improve tissue response to weight loss interventions.

\section{Funding Sources}

This work was supported by Agence Nationale de la Recherche (ANR MICRO-Obes, and ANR-10-IAHU-05) and the European Union's Seventh Framework Programme for research, technological development and demonstration under grant agreement HEALTHF4-2012-305312 (METACARDIS). The clinical work received support from KOT-Ceprodi, Danone Nutricia Research and the Foundation Coeur et Artère.

\section{Influence of Vitamin D on Neurodegenerative Candidate Gene Expression (OR20-01)}

Joe L Webb and Matthew Rowling

Iowa State University

Objectives: Vitamin D insufficiency correlates with increased risk of dementia, and genome-wide association studies examining neurologic diseases have identified numerous candidate genes that may be modified by dietary factors such as vitamin D. This study had the following 2 objectives: 1 ) to assess the effects of dietary whole egg, a rich source of vitamin D, on expression of genes associated with Alzheimer's and Parkinson's disease in nervous tissues of Zucker diabetic fatty $(\mathrm{ZDF})$ lean control $(\mathrm{FA} /+)$ rats; and 2) to assess the effects of 1,25-dihydroxycholecalciferol $\left[1,25(\mathrm{OH})_{2}-\mathrm{D}_{3}\right]$ on expression of genes associated with Alzheimer's and Parkinson's disease through the use of mixed neuronal and glial cell cultures.

Methods: ZDF lean control rats $(n=16)$ were randomly assigned to either an AIN93 casein-based diet, or a modified diet supplemented with whole egg. Protein was provided at $20 \%(\mathrm{w} / \mathrm{w})$ of the diets and diets were matched for lipid content (18.3\%) by adding corn oil to the casein-based diets to account for additional lipid in whole egg. The transcriptomic response of hippocampal tissues from rats was assessed with the use of quantitative polymerase chain reaction (qPCR). Similarly, the transcriptomic response of neuronal cell cultures treated with either $1 \times 10^{-8} \mathrm{M} 1,25(\mathrm{OH})_{2}-\mathrm{D}_{3}$ or ethanol vehicle as a control was assessed by qPCR. Gene expression was conducted on a Light Cycler 96 and primers were designed with the use of IDT's PrimerQuest Tool. Data were analyzed with the use of the deltadelta-CT method and presented as fold changes between treatment groups.

Results: Whole egg consumption in rats modified expression of vitamin $\mathrm{D}$ receptor (VDR), triggering receptor expressed on myeloid cells (TREM) 2, TREM1, NF- $\kappa$ B, and IL6; however, parkin RBR E3 ubiquitin protein ligase (PRKN), protein tyrosine kinase 2 beta (PTK2B), and solute carrier family 24 member 4 (SLC24A4) did not show significantly different changes in gene expression. Most strikingly, nuclear receptor related 1 (NURR1) expression decreased 20 -fold in rats fed a whole egg-based diet. Vitamin D administration to culture modified expression of bridging integrator 1 (BIN1), clusterin (CLU), NURR1, and prokineticin 2 (PROK2); and surprisingly ATP-binding cassette A7 (ABCA7) expression increased 5 -fold.

Conclusion: These data support the hypothesis that dietary consumption of whole egg has the potential to modify expression of Alzheimer's and Parkinson's disease genes, which may be related to the vitamin $\mathrm{D}$ content in whole egg. In support of this theory, administration of $1,25(\mathrm{OH})_{2}-\mathrm{D}_{3}$ to mixed neuronal-glial cell cultures also modifies expression of these genes.

\section{Funding Sources}

This work was supported, in part, by a National Science Foundation Graduate Research Fellowship, grants from the Alfred P Sloan Foundation G-2014-13746, National Science Foundation NSF ACI 1349002.

Differential Expression and Coexpression Analyses on Transcriptional Profiles of Lower Small Intestine Suggest Key Pathways Regulated by Vitamin A (OR20-02)

Zhi Chai, Yafei Lyu, Qiuyan Chen, Cheng-Hsin Wei, Lindsay Snyder, Veronika Weaver, Qunhua Li, Margherita Cantorna, and Catharine Ross

\section{Pennsylvania State University}

Background: Vitamin A (VA) deficiency remains a public health issue in resource-limited areas. It is estimated that $>20 \%$ of preschoolaged children have clinical VA deficiency. Diarrheal-related diseases are 
a major cause of mortality in VA-deficient children. Citrobacter rodentium is a natural mouse enteropathogen mimicking enteropathogenic Escherichia coli (EPEC) infection in humans. During C. rodentium infection, vitamin A-sufficient (VAS) mice displayed a better survival rate and enhanced pathogen clearance compared with their vitamin A-deficient (VAD) counterparts.

Objective: The aim of this study was to survey the transcriptomes in lower small intestine (SI), and to identify novel biological pathways regulated by VA that protect the host against $C$. rodentium infection.

Methods: VA deficiency was induced in C57/BL6 mice by feeding VAD diet to the pregnant dam and their postweaning offspring. Age-matched VAD or VAS mice were orally inoculated with $C$. rodentium (infected group) or phosphate-buffered saline only (noninfected group). Lower SI samples were collected $5 \mathrm{~d}$ postinfection for RNAseq measurements on a Illumina HiSeq 2500 platform. Differential expression, coexpression network, and functional enrichment analyses were performed on the RNAseq data.

Results: Differentially expressed genes (DEGs) were detected between the VAS and VAD groups, 5.6\% of which are related to transcription regulation and $18.4 \%$ to immune response. The DEG list contains retinoic acid target genes that are known players in retinol metabolism or mucosal immunity. Coexpression modules were identified based on the top 2000 DEGs. Functional enrichment in immune system process and chemotaxis were found in $55 \%$ of the modules. Two modules were highlighted for being enriched in the category of leukocyte transendothelial migration, indicating that VA deficiency altered both the leukocytes and the endothelial cells in the SI. The network of each module was visualized to reflect the adjacencies between gene pairs and the weight of each module members.

Conclusions: The results showed that VA deficiency significantly affected innate and adaptive immune responses in the SI. Leukocyte transendothelial migration may be a novel mechanism under the regulation of VA in the SI.

Funding Sources

NIH grants T32 GM108563 and R56 AI114972.

Epigenetic Control of Cortical Estrogen Receptor (Er)- $\alpha$ is Altered by Early-Life Bisphenol A and High-Fat Diet Exposure Differentially by Sex (OR20-03)

Diego Hernandez-Saavedra, Leslie Wise, Janice Juraska and Yuan-Xiang Pan

University of Illinois Urbana-Champaign

Background: Early-life exposure to high-fat (HF) diet and endocrine disruptors such as bisphenol A (BPA) directly impact behavior and metabolism partly by altering the epigenetic landscape. The prefrontal cortex (PFC) is of importance given its vital role in executive function, memory, and intelligence. Behavioral alterations seen in animals exposed to BPA or an HF diet at early developmental stages might be explained by changes in estrogen receptor $(E r)-\alpha$ in PFC, which could impact estrogen sensitivity later in life.

Objective: The aim of this study was to evaluate the genetic and epigenetic effect of early-life BPA and/or HF exposure on $E r-\alpha$ in the $\mathrm{PFC}$ of male and female rats.
Methods: From gestational day 2 through parturition, female Long Evans hooded dams were assigned to either a control (CON, 15.8\% $\mathrm{kcal}$ fat) or HF diet ( $45 \% \mathrm{kcal}$ fat). Concurrently, BPA doses were administered at $0,40,400 \mu \mathrm{g} \mathrm{BPA} / \mathrm{kg}$ body weight in a cookie to the dams, or via oral dosing to the pups until postnatal day (P) 10. After $\mathrm{P} 10$, animals consumed the CON diet with no additional BPA dosing. At P10 and P90, offspring animals were killed and the PFC was collected for mRNA, DNA methylation, and microRNA analysis.

Results: Overall, Er- $\alpha$ expression in PFC was increased by the HF $\operatorname{diet}(P=0.03)$ and BPA (400 $\mu \mathrm{g}, P=0.04)$; at P10, Er- $\alpha$ was increased in females $(P=0.05)$ and a 400 BPA dose $(P=0.001)$, whereas at P90, the HF diet increased $E r-\alpha(P=0.03)$ and males with a $400 \mathrm{BPA}$ dose decreased $E r-\alpha(\mathrm{P}=0.03)$. Analysis of DNA methylation patterns of 4 regions (regions 1-4) of $E r-\alpha$ promoter revealed a decrease in region 1 for females consuming the HF $\operatorname{diet}(P=0.014)$, and a decrease in region 4 by HF diet $(P=0.049)$; however, no active transcription changes were detected by $E r-\alpha$ premRNA expression. Identification of microRNA though database mining retrieved 3 conserved miRNAs that target Er- $\alpha$ (mir-19b-3p, mir-221/222-3p, mir-22-3p), which showed significant Sex $\times$ BPA and BPA $\times$ miRNA interactions with $E r-\alpha$ expression.

Conclusion: Early-life exposure to BPA or HF diet regulated gene expression of $E r-\alpha$ in PFC in a sex-dependent manner, not by altering its DNA methylation pattern, but by inducing the expression of small microRNAs that target $E r-\alpha$ for degradation that could alter executive function and cognition later in life.

\section{Funding Sources}

P01 ES002848-Project 3 and USEPA 83543401 Project 3.

\section{Effects of Eicosapentaenoic Acid in High-Fat-Fed UCP1- Deficient Male Mice (OR20-04)}

Mandana Pahlavani, Latha Ramalingam, Emily Miller, Iurii

Koboziev, Shane Scoggin, and Naima Moustaid-Moussa

Texas Tech University

Objectives: Activation of brown adipose tissue (BAT) and browning of white adipose tissue (WAT) have emerged recently as potential means to increase energy expenditure and reduce obesity. The thermogenic function of BAT is highly dependent on uncoupling protein 1 (UCP1). We have previously reported that eicosapentaenoic acid (EPA) significantly increased BAT UCP1 protein as well as expression of several other master regulators of thermogenesis. The precise mechanisms for BAT activation by these dietary fatty acids remains unknown. We hypothesize that these beneficial metabolic effects of EPA require UCP1 expression.

Methods: We used wild-type (WT) and UCP1 knockout (KO) B6 male mice. The $\mathrm{KO}$ mice were housed in a thermoneutral environment $\left(30^{\circ} \mathrm{C}\right)$, previously reported to induce obesity in $\mathrm{KO}$ mice. All mice were fed a high-fat diet (HF, 45\% kcal fat) for up to $14 \mathrm{wk}$, either without (HF) or with (EPA) $36 \mathrm{~g} / \mathrm{kg}$ of fish oil (800 mg EPA/g fish oil) kindly provided by Organic Technologies. We measured body weight, body composition, food intake, insulin, and glucose tolerance during the feeding period. Tissues were fixed for histologic studies and also frozen for later analyses.

Results: WT groups (both HF and EPA) consumed significantly more food than the KO groups $(P<0.05)$. However, the body weights 
of both WT groups were significantly lower $(P<0.05)$ compared with their respective $\mathrm{KO}$ groups. EPA significantly improved glucose clearance and insulin sensitivity in WT and KO mice, compared with mice fed HF diets, indicating protective effects of EPA. Furthermore, both WT and KO mice fed EPA showed significantly lower WAT and BAT weights, consistent with lower total body fat percentage compared with their respective counterparts. The BAT slides from histology sections in WT mice showed reduced lipid content, compared with WT mice fed HF, whereas no differences were observed between the HF and EPA diets in the KO mice.

Conclusions: These results validate the beneficial effects of EPA in energy homeostasis and insulin resistance, which appear to be independent of UCP1. However, changes in lipid content in BAT with EPA may be dependent on UCP1. Further cellular analyses that used tissues and stem cells from WAT and BAT from both genotypes are warranted to dissect the mechanisms mediating EPA and UCP1 effects on energy homeostasis and lipid metabolism.

Funding Sources

NIH/NCCIH grant R15AT008879-01A1.

CKM Genetic Variants and Muscle Vulnerability to Low Magnesium Intake (OR20-05)

\section{Martin Kohlmeier ${ }^{1}$ and Olivia Dong ${ }^{2}$}

${ }^{1}$ University of North Carolina Nutrition Research Institute; and ${ }^{2}$ University of North Carolina at Chapel Hill

Objective: Data from the very large UK Biobank cohort confirmed earlier observations that low magnesium intake can weaken muscle health. The effects are small and may be most significant in a subgroup of people. We wanted to know whether the common variants in the CKM gene coding for the magnesium-dependent creatine kinase in skeletal muscle affect the response to magnesium intake.

Methods: Responses to a single bout of eccentric elbow flexor exercise were examined before and $3 \mathrm{~d}$ after the challenge in 58 healthy men by measuring creatine kinase (CK) activity and by assessing muscle performance with a dynamometer (Biodex 3). Dietary intakes were assessed by ASA24 interviews on $3 \mathrm{~d}$ under close dietitian guidance. The CKM gene was analyzed with an Illumina MEGA-Ex chip.

Results: We used CK activity $3 \mathrm{~d}$ after exercise (AE) to assess the impact of $C K M$ variants in a $50-\mathrm{kb}$ window including previously reported CK-linked loci. The variant rs344816 (a proxy for the nonsynonymous exonic variant rs344815) was most closely associated with AE CK activity ( $r=0.382,>4$ times CK reference range). A statistically significant inverse correlation of magnesium intake and $\mathrm{AE}$ CK activity was seen in men with 0 or 1 rs344816 A alleles $(r=0.304$, $P=0.04)$, but not in those with 2 A alleles $(r=0.072)$. An asyet unexplained finding is that the 13 carriers of the rs344816 AA genotype had much higher magnesium intakes than men with the other genotypes ( 458 compared with $344 \mathrm{mg} / \mathrm{d}, P<0.01$ ). None of them had intakes $<300 \mathrm{mg} / \mathrm{d}$, although 4 of the 16 men without an A allele did have such very low intakes.
Conclusions: We conclude that the CKM rs344816 A allele increases healthy men's muscle vulnerability to eccentric exercise and that low magnesium intake further increases risk.

\section{Funding Sources}

UNC Nutrition Research Institute.

Maternal Plasma Polyunsaturated Fatty Acid Concentrations during Pregnancy and Cord Blood DNA Methylation: An Epigenome-Wide Association Study (OR20-06)

Yu-Han Chiu, ${ }^{1}$ Andres Cardenas, ${ }^{2}$ Sheryl L Rifas-Shiman, ${ }^{2}$ Marie-France Hivert, ${ }^{2}$ Dawn L DeMeo, ${ }^{3}$ Augusto Litonjua, ${ }^{4}$ Andrea A Baccarelli, ${ }^{5}$ Emily Oken, ${ }^{2}$ and Jorge Chavarro ${ }^{1}$

${ }^{1}$ Harvard TH Chan School of Public Health, MA; ${ }^{2}$ Harvard Medical School and Harvard Pilgrim Health Care Institute, MA; ${ }^{3}$ Brigham and Women's Hospital and Harvard Medical School, MA; ${ }^{4}$ University of Rochester Medical Center, NY; and ${ }^{5}$ Department of Environmental Health Sciences

Objectives: Omega-3 (n-3) and omega- $6(n-6)$ polyunsaturated fatty acids (PUFAs) play an important role in fetal growth and postnatal development. DNA methylation, which undergoes dynamic changes during fetal development, may be one of the underlying mechanisms. We investigated the associations of maternal PUFA concentrations measured in midpregnancy with epigenome-wide DNA methylation in cord blood nucleated cells in Project Viva, a prospective prebirth cohort in Massachusetts.

Methods: We measured plasma PUFA concentrations by gas liquid chromatography, and genome-wide methylation levels in cord blood cells with the use of HumanMethylation450 Bead Chips (at 373,563 CpG sites after quality control) among 259 mother-infant pairs. We conducted robust linear regressions adjusting for cord blood cell types, maternal age, prepregnancy body mass index, parity, education, gestational age at delivery, child sex, and race, with a false discovery rate $<0.05$ as a significance level.

Results: The mean \pm SD plasma total $\omega-6$ and $\omega-3$ concentrations in midpregnancy were $1422 \pm 507$ and $134 \pm 56 \mu \mathrm{g} / \mathrm{mL}$, respectively. Maternal total $\omega$-6 levels were associated with higher DNA methylation at CpG cg16327228 ( $\beta$ : 0.038\%; 95\% CI: $0.025 \%, 0.052 \%$ per $1 \mu \mathrm{g} / \mathrm{mL}$ increase in total $\alpha-6$ concentration) annotated to Mohawk homeobox $(M K X)$, a gene involved in cell adhesion and tendon development. This association was mainly driven by linoleic acid. When individual $\omega-6$ levels were analyzed separately, we further identified two CpG sites associated with arachidonic acids: $\operatorname{cg} 12124589$ ( $\beta$ : 0.07\%; 95\% CI: $0.04 \%, 0.09 \%)$ annotated to Quiescin Sulfhydryl Oxidase 1 (QSOX1) and $\operatorname{cg} 25661142(\beta$ : $-0.13 \%$; 95\% CI: $-0.18 \%,-0.09 \%)$ annotated to Widely Interspaced Zinc Finger Motifs (WIZ), both of which are involved in growth development. We did not identify any CpG sites that were differentially methylated according to maternal total $\omega-3$ concentrations.

Conclusions: Prenatal $\omega-6$ concentrations were associated with newborn DNA methylation at $\mathrm{CpG}$ sites annotated to genes involved in fetal development. 
Supporting Images/Graphs
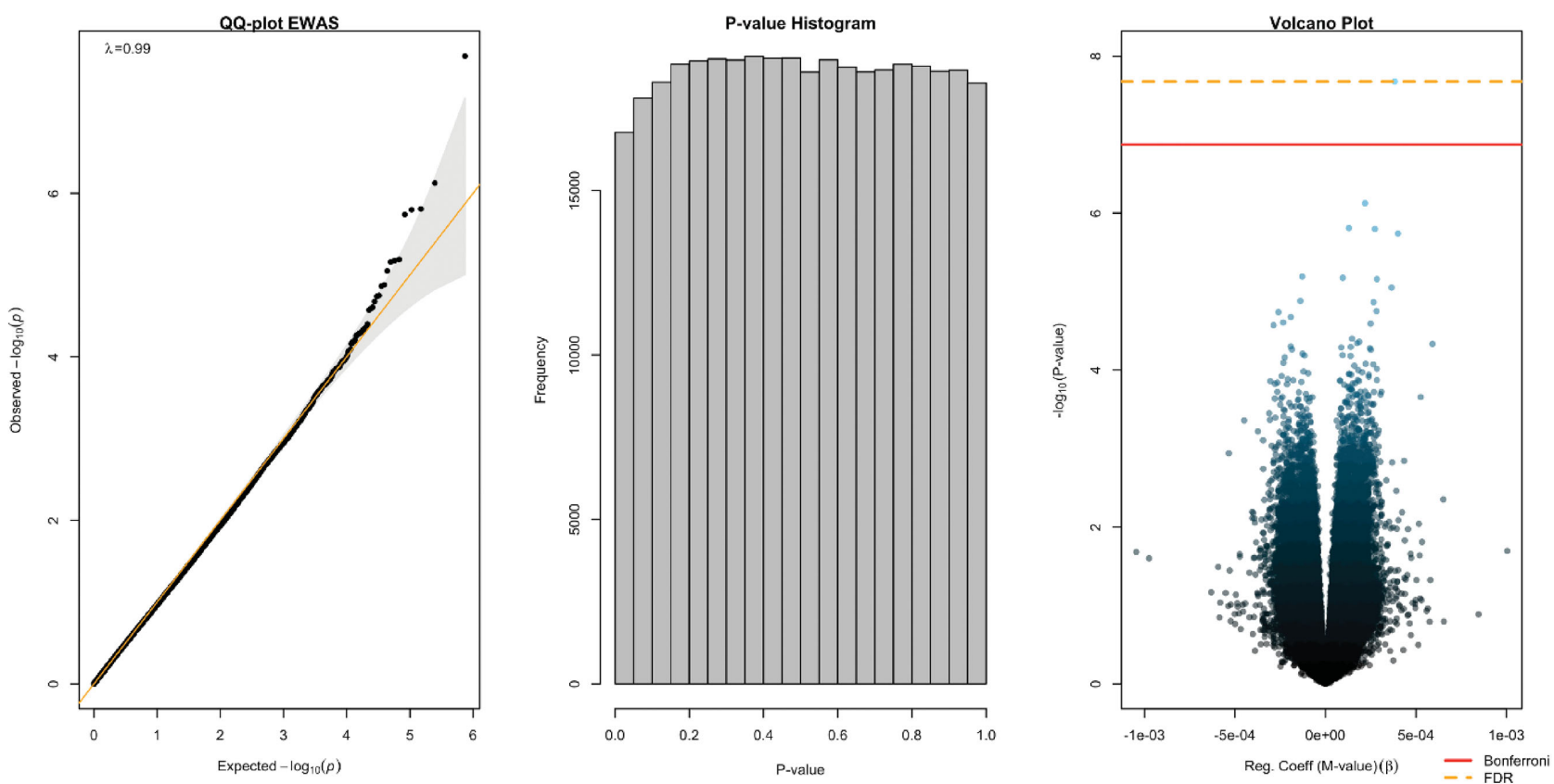

FIGURE OR20-06-1 EWAS of 2nd trimester plasma total omega-6 fatty acids and cord blood DNA methylation

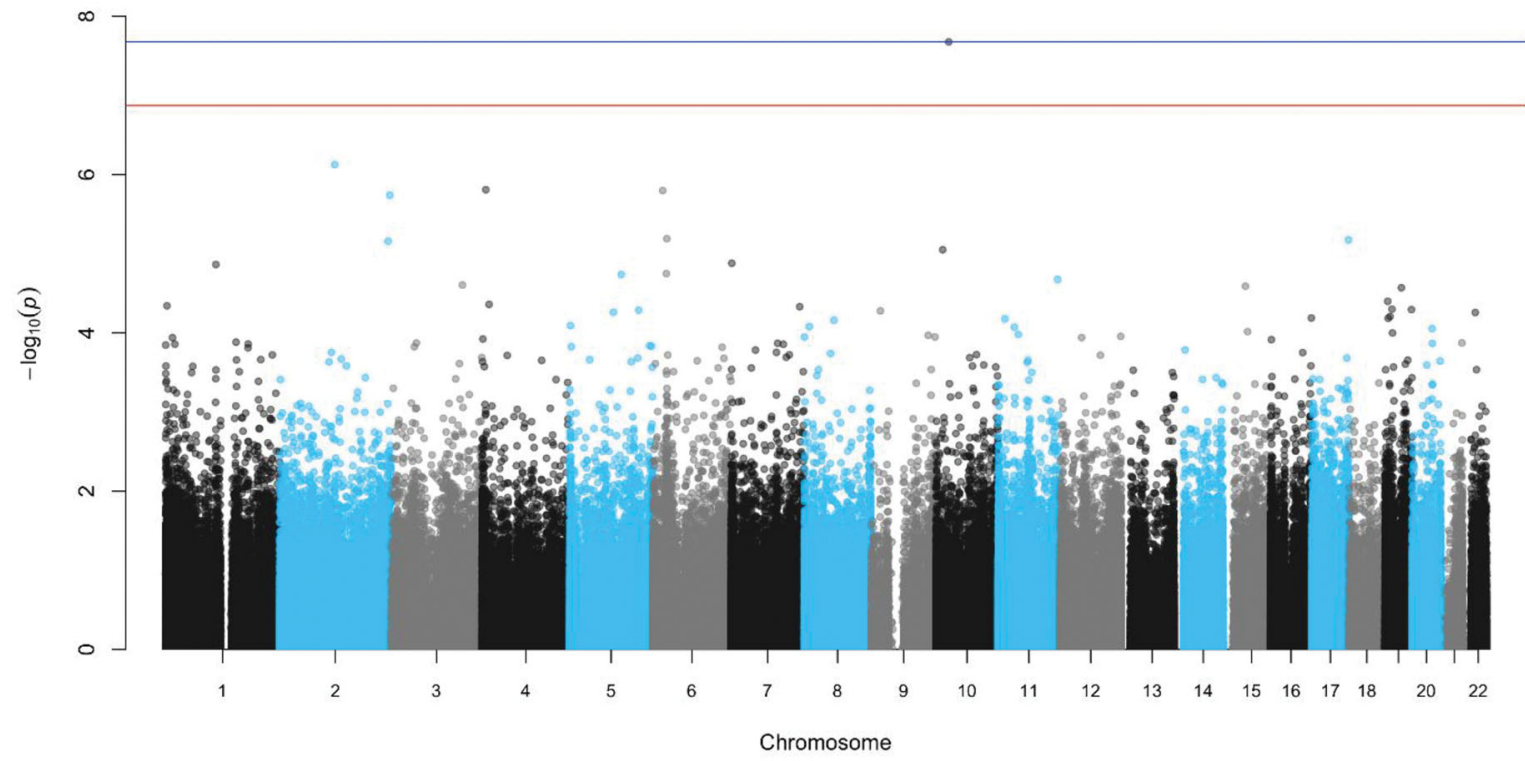

FIGURE OR20-06-2 Manhattan plot of 2nd trimester plasma total omega-6 levels and cord blood DNA methylation

\section{Funding Sources}

$\mathrm{NIH}$ grants R01 HD 03,4568, HL111108, K24HD069408,

UG3OD023286, P30DK046200, P30ES00002, and K23ES024803. 
Carbohydrate Intake Drives CpG Methylation at CPT1A Affecting Risk of Metabolic Diseases (OR20-07)

Chao-Qiang Lai, ${ }_{1}^{1}$ Carl Bender, ${ }^{2}$ Stella Aslibekyan, ${ }^{3}$ Tao Guo, ${ }^{4}$ Laurence D Parnell, ${ }^{4}$ Hemant Tiwari, ${ }^{3}$ Sergi Sayols-Baixeras, ${ }^{5}$ Marguerite R Irvin, ${ }^{3}$ David Fei, ${ }^{4}$ Bertha A Hidalgo, ${ }^{3}$ Paul N Hopkins, ${ }^{5}$ Devin Absher, ${ }^{6}$ Michael Province, ${ }^{7}$ Roberto Elosua, ${ }^{8}$ Donna K Arnett, ${ }^{9}$ and Jose M Ordovas ${ }^{4}$

${ }^{1}$ USDA Agricultural Research Service; ${ }^{2}$ Tufts University, MA; ${ }^{3}$ School of Public Health, University of Alabama; ${ }^{4}$ Jean Mayer USDA Human Nutrition Research Center on Aging at Tufts University, MA; ${ }^{5}$ University of Utah; ${ }^{6}$ HudsonAlpha Institute for Biotechnology, AL; ${ }^{7}$ Washington University School of Medicine, MO; ${ }^{8}$ IMIM-Hospital del Mar Medical Research Institute, Spain; and ${ }^{9}$ College of Public Health, University of Kentucky

Objective: Dietary habits can modify epigenetic status, which affects the risk of the metabolic diseases. A recent epigenome-wide association identified the cg00574958 DNA methylation site at CPT1A, which is associated with body mass index (BMI), triglyceride (TG), and glycemic traits, but what causes this association is unknown.

Methods: We examined associations between carbohydrate and total fat intake, normalized to total energy intake, and the carbohydrate: fat ratio with methylation site CPT1A-cg00574958, and obesity, fasting TG, and glucose measures in 3 populations (Genetics of Lipid Lowering Drugs and Diet Network, $n=978$; Framingham Heart Study, $n=2331$; REgistre GIroní del COR study, $n=645$ ), adjusting for potential cofounding factors: age, sex, smoking, alcohol use, medication, physical activity, and family relations. To determine the causal effect of dietary intake on the risk of metabolic diseases, we performed meta-analysis and Mendelian randomization analysis based on a random-effect model with carbohydrate and fat intakes as exposures and cg00574958 as the instrument variable.

Results: We showed that carbohydrate intake and the carbohydrate:fat ratio were positively associated with cg00574958 methylation, whereas fat intake was negatively correlated with cg00574958 methylation level. Meta-analysis further confirmed this strong correlation with $\beta=58.4, P=8.98 \times 10^{-16}$ for carbohydrate intake, $\beta=-36.4$ and $P=9.96 \times 10^{-10}$ for total fat intake, and $\beta=3.30$ and $P=1.48 \times 10^{-11}$ for the carbohydrate:fat ratio. Furthermore, cg00574958 was strongly correlated with metabolic phenotypes (BMI, TG, glucose) in all 3 populations. Mendelian randomization illustrated that the carbohydrate:fat ratio had a causal effect on TG $(\beta=-2.41$; 95\% CI: $-3.67,-1.14 ; \mathrm{P}=0.0002)$, and total fat intake on glucose concentration ( $\beta=0.056$; 95\% CI: 0.016, 0.096; $\mathrm{P}=0.006$ ). However, carbohydrate intake alone was not causal with respect to the risk of metabolic diseases.

Conclusions: Our results reveal that the ratio between carbohydrate and total fat intake has a causal effect on CpG methylation.

\section{Funding Sources}

This work was funded by the US Department of Agriculture, agreement no. 8050-51000-098-00D, and by National Heart, Lung and Blood Institute grants U01-HL072524-04 and R01HL104135. SSB was funded by a contract from Instituto de Salud Carlos III-FEDER (IFI14/00007).
Effects of Canola and High-Oleic-Acid Canola Oils on Expression of Genes Related to Lipid and Lipoprotein Metabolism in Human Peripheral Blood Mononuclear Cells (OR20-08)

Kate J Bowen, ${ }^{1}$ Todd Rideout, ${ }^{2}$ Peter JH Jones, ${ }^{3}$ Phillip W Connelly, ${ }^{4}$ Patrick Couture, ${ }^{5}$ David Jenkins, ${ }^{4}$ Benoit Lamarche, ${ }^{5}$ Carla G Taylor, ${ }^{3}$ Sheila G West, ${ }^{1}$ and Penny M Kris-Etherton ${ }^{1}$

${ }^{1}$ Pennsylvania State University; ${ }^{2}$ University at Buffalo, NY; ${ }^{3}$ University of Manitoba, Canada; ${ }^{4}$ University of Toronto, Canada; and ${ }^{5}$ Laval University, Canada

Objectives: We have demonstrated that canola and high-oleicacid canola oils (HOCOs) improve circulating atherogenic lipids and lipoproteins compared with a control oil with a fatty acid profile characteristic of Western intakes; however, the molecular mechanisms underlying these diet-induced clinical responses are unknown. The purpose of this study was to utilize a nutrigenomic approach to investigate the expression of targeted lipid and lipoprotein-related genes to elucidate the biological mechanisms. We hypothesized that canola oil and HOCO cause differential expression in genes regulating lipid and lipoprotein metabolism compared with a control oil.

Methods: In a double-blind, randomized, 3-period crossover, multisite, controlled feeding trial, participants with an increased waist circumference plus at least one additional criterion for metabolic syndrome consumed 3 isocaloric, weight-maintenance diets for $6 \mathrm{wk}$ separated by $\geq 4$-wk washouts: 1 ) a canola oil diet [17.5\% of energy from monounsaturated fatty acids (MUFAs), 9.2\% polyunsaturated fatty acids (PUFAs), 6.6\% saturated fatty acids (SFAs)]; 2) a HOCO diet (19.1\% MUFA, 7.0\% PUFA, 6.4\% SFA); and 3) a control oil diet (10.5\% MUFA, $10.0 \%$ PUFA, $12.3 \%$ SFA). Blood was collected at the end of each diet period in PAXgene Blood RNA tubes from a subset of participants $(n=50)$. Expression of 21 genes regulating lipid and lipoprotein metabolism was measured in peripheral blood mononuclear cells (PBMCs) by real-time polymerase chain reaction analysis. Data were normalized to $\beta$-actin.

Results: Canola oil and HOCO increased expression of ATPbinding cassette transporter $(\mathrm{ABC}) \mathrm{A} 1$ (canola: 43.9\%, $P=0.0005$; HOCO: $37.6 \%, P=0.0041$ ) and ABCG1 (canola: $17.4 \%, P=0.0023$; HOCO: $17.4 \%, P=0.0022$ ) in PBMCs compared with control. There was no significant treatment effect on endpoint expression of the other 19 genes.

Conclusions: Canola oil and HOCO diets increased expression of genes implicated in regulation of cholesterol efflux in human PBMCs compared with a diet with a fatty acid profile reflecting Western intakes. These data indicate that dietary fatty acid composition modifies gene transcription, and suggest that consumption of vegetable oils high in unsaturated fatty acids may confer antiatherogenic and antiinflammatory benefits by promoting cholesterol efflux pathways.

\section{Funding Sources}

This research is part of the Canola Agri-Science Cluster, with funding provided by Agriculture and Agri-Food Canada, the Canola Council of Canada, Alberta Canola, SaskCanola, Dow Agro Sciences, and the Manitoba Canola Growers. The research was also supported by National Center for Research Resources grant UL1 RR033184, which is now at the National Center for Advancing Translational Sciences grant UL1 TR000127, as well as NIH grant 2T32DK007703. 
Mechanisms Mediating Reversal of Liver Steatosis by Eicosapentaenoic Acid: Studies in High-Fat-Diet-Induced Obese Mice and in HepG2 Hepatoma Cells (P17-001)

Kembra D Albracht-Schulte, ${ }^{1}$ Samantha Gonzalez, ${ }^{1}$ Abigail Jackson, ${ }^{1}$ Latha Ramalingam, ${ }^{1}$ Nishan Kalupahana, ${ }^{2}$ and Naima Moustaid-Moussa ${ }^{1}$

${ }^{1}$ Texas Tech University; and ${ }^{2}$ University of Peradeniya, Sri Lanka

Objective: Nonalcoholic fatty liver disease (NAFLD) is increasing in prevalence worldwide due to rising obesity rates. NAFLD is referred to as the hepatic manifestation of the metabolic syndrome. Omega-3 (n-3) polyunsaturated fatty acids, such as eicosapentaenoic acid (EPA), have anti-inflammatory and triglyceride-lowering properties and may help reduce NAFLD. We have previously reported that EPA prevented and reversed high-fat (HF)-diet-induced obesity, insulin resistance, and hepatic steatosis in B6 mice. Interestingly, reductions in hepatic triglycerides with EPA were independent of weight status. However, the mechanisms underlying these improvements are not clear. We hypothesized that EPA reduced fatty liver by increasing fat oxidation, and reducing lipogenesis and inflammation, in part through microRNA (miRNA)-mediated mechanisms.

Methods: We analyzed gene, protein, miRNA, and metabolic changes in liver samples from B6 mice fed HF diets, without (HF), or with EPA (HF-EPA). We further tested direct effects of EPA and other fatty acids on HepG2 gene and miRNA expression and energy metabolism with the use of a Seahorse Extracellular Flux Analyzer.

Results: Hepatic lipogenesis was decreased in HF-EPA mice, as indicated by decreased protein and mRNA levels of fatty acid synthase (FAS) and acetyl-CoA carboxylase (ACC). With EPA, liver metabolism shifted towards $\beta$-oxidation, as indicated by increased gene expression of peroxisome proliferator-activated receptor- $\alpha$ (PPAR $\alpha)$, and carnitine palmitoyltransferase 1a and 2. Additionally, inflammation was reduced, as shown by decreased tumor necrosis factor $\alpha$ gene expression. Accordingly, EPA also significantly reduced FAS and ACC mRNAs in HepG2 cells. Glycolysis, estimated by extracellular acidification rate, was significantly reduced in HepG2 cells treated with EPA compared with vehicle. Furthermore, we identified several miRNAs that are regulated by EPA in mouse liver. Among these, EPA significantly increased hepatic miR-let7a-5p, which targets inflammatory pathways.

Conclusions: EPA regulates hepatic lipid-, carbohydrate-, and inflammation-related processes. These changes may be mediated by specific miRNAs. Our findings provide novel mechanistic evidence for beneficial effects of EPA in NAFLD.

\section{Funding Sources}

KAS is a predoctoral fellow supported by the National Institute of Food and Agriculture, US Department of Agriculture, AFRI ELI Predoctoral Fellowship, under award number 2017-67011-26029.

Autonomous Modulation of Skeletal Muscle Remodeling and Energy Metabolism Gene Sets by the Vitamin D receptor (P17-003)

Joseph J Bass, ${ }^{1}$ Daniel Wilkinson, ${ }^{1}$ Kenneth Smith, ${ }^{1}$ Andrew Philp, ${ }^{2}$ Nathaniel J Szewczyk, ${ }^{1}$ Mark Cleasby, ${ }^{3}$ Iain J Gallagher, ${ }^{4}$ and Philip J Atherton ${ }^{1}$
${ }^{1}$ University of Nottingham, United Kingdom; ${ }^{2}$ University of Birmingham, United Kingdom; ${ }^{3}$ Royal Veterinary College, United Kingdom; and ${ }^{4}$ University of Stirling, United Kingdom

Objective: Vitamin D (VitD) deficiency is prevalent in $\sim 25 \%$ of the population, and is proposed to have actions upon skeletal muscle metabolism and health. VitD regulates and exerts its effects through the ubiquitously expressed VitD receptor (VDR), a potent transcription factor that controls the expression of multiple genes through modulation of Pol II preinitiation complexes. Recent investigations have established an autonomous role for VDR in muscle function and regeneration. Thus, we aimed to investigate whether VDR has a functional role in modulating key regulatory pathways/gene sets important to muscle growth and homeostasis.

Methods: To examine the role of VDR in muscle mass regulation, tibialis cranialis (TC) muscles of Wistar rats were electroporated (under $2.5 \%$ isofluorane, $50 \mathrm{mg}$ carprofen $/ \mathrm{kg}$ ) to continuously overexpress (VDR-OE) or knockdown (VDR-KD) VDR by cDNA or shRNA lentiviral transfection; contralateral TCs were sham-treated internal controls. RNA from muscles underwent RNA-Seq with the Illumina HiSeq system, before analysis of differential gene expression and gene set enrichment. False discovery rates were applied throughout.

Results: VDR-OE stimulated fiber hypertrophy [cross-sectional area (CSA) $+17 \% \pm 7 \%, P<0.05$ ] and enhanced muscle protein synthesis $(+69 \% \pm 7 \%, P<0.05)$. This was matched by an upregulation in key growth-related extracellular remodeling and integrin pathway gene sets. Comparatively, VDR-KD induced myofiber atrophy (CSA $-8 \% \pm 2 \%, P<0.001$ ) and autophagy-related processes (e.g., LC3BII $+84 \% \pm 43 \%, P<0.05)$. This was complemented by an upregulation in multiple lysosome and vesicle biogenesis gene sets. Moreover, many energy metabolism-related genes were downregulated, i.e., electron transport, oxidative phosphorylation, glycolysis, and citric acid cycle gene sets.

Conclusions: The VDR acts autonomously to regulate muscle mass, operating through alternate gene set modulation to facilitate hypertrophy through enhanced remodeling, and limiting atrophy by regulating energy metabolism and autophagy.

Funding Sources

JJB was funded by the MRC doctoral training programme (MR/J500495/1).

Dark Chocolate Consumption Can Differentially Modulate Human Gene Expression for $T$ Cell Activation, Cellular Immune Response, Neural Signaling, and Sensory Perception (P17-004)

Lee Berk, ${ }^{1}$ Kristin Bruhjell, ${ }^{1}$ Warren Peters, ${ }^{1}$ Peter Bastian, ${ }^{1}$ Everett Lohman, ${ }^{1}$ Gurinder Bains, ${ }^{1}$ Jesusa Arevalo, ${ }^{2}$ and Steve Cole $^{2}$

${ }^{1}$ Loma Linda University Health, CA; and ${ }^{2}$ UCLA School of Medicine, CA

Objective: The aim of this study was to map the impact of $70 \%$ cocoa consumption on human immune and dendritic cell gene expression, focusing on pro/anti-inflammatory cytokines.

Methods: This pilot study enrolled 4 subjects ( 2 female/ 2 male, aged 25-50 y) and 1 control (female, 28 y). After a baseline 
blood draw, subjects consumed $70 \%$ cocoa over 10 min [antioxidant activity (aa), 46,000 $\mu \mathrm{mol}$ Trolox equivalent (TE)/100 g]. Follow-up samples were obtained after $2 \mathrm{~h}$ and 1 wk. Subjects consumed $48 \mathrm{~g} 70 \%$ cocoa/day ( 2 pieces/h; aa $3834 \mu \mathrm{mol} \mathrm{TE} / 100$ g). Total RNA was assayed by RNAseq, with data analyzed by linear models to identify differentially expressed genes, followed by bioinformatic analyses of transcription control pathways and cellular origins.

Results: Leukocyte transcriptome profiles showed little systematic change in the initial $2 \mathrm{~h}$ of cocoa exposure, although prespecified bioinformatics analyses indicated small increases in activity of genes regulated by $\mathrm{NF}-\kappa \mathrm{B}$ and $\mathrm{AP} 1$ transcription factors and $\mathrm{CD} 4+$ and CD8+ T cells (all $P<0.05$ ). By contrast, analyses of change from baseline to 1 wk identified 177 genes showing significant differential expression through the use of an a priori-specified false discovery rate cut-point of 0.20 (140 upregulated, 37 downregulated), with 116 achieving $q<0.05$. Upregulated genes were involved in leukocyte activation and motility (CD40, TICAM2, AZU1, HRAS and RASD2, HSPA1L, FGF22); derived from CD4+ and CD8+ T lymphocytes
$(P<0.001$ and 0.0148$)$; and showed overrepresentation of promoter elements for NF- $\kappa$ B, SP-1, MZF-1, and HIF-1 transcription factors (all $P<0.01)$ and underrepresentation of AP-1 $(P=0.018)$. Upregulated transcripts also included genes involved in neural signaling and sensory perception (OR2J3, OR52A5, OR6C3, OR9I1, NR4A2, and NRXN2).

Conclusion: Cocoa consumption upregulates multiple intracellular signaling pathways involved in $\mathrm{T}$ cell activation and cellular immune response. The results are consistent with previous indications that cocoa components inhibit MAPK activation, but suggest $\mathrm{T}$ cell function may be preserved and enhanced via other compensatory molecular pathways. It should be noted that the NF- $\kappa$ B upregulation source was not from upregulated monocytes, suggesting a role other than cascading inflammation. It is of interest to note that genes involved in neural signaling and sensory processes were upregulated.

\section{Funding Sources}

Departmental.

Supporting Images/Graphs

Cocoa \& Gene Expression

\section{Cellular Origin}

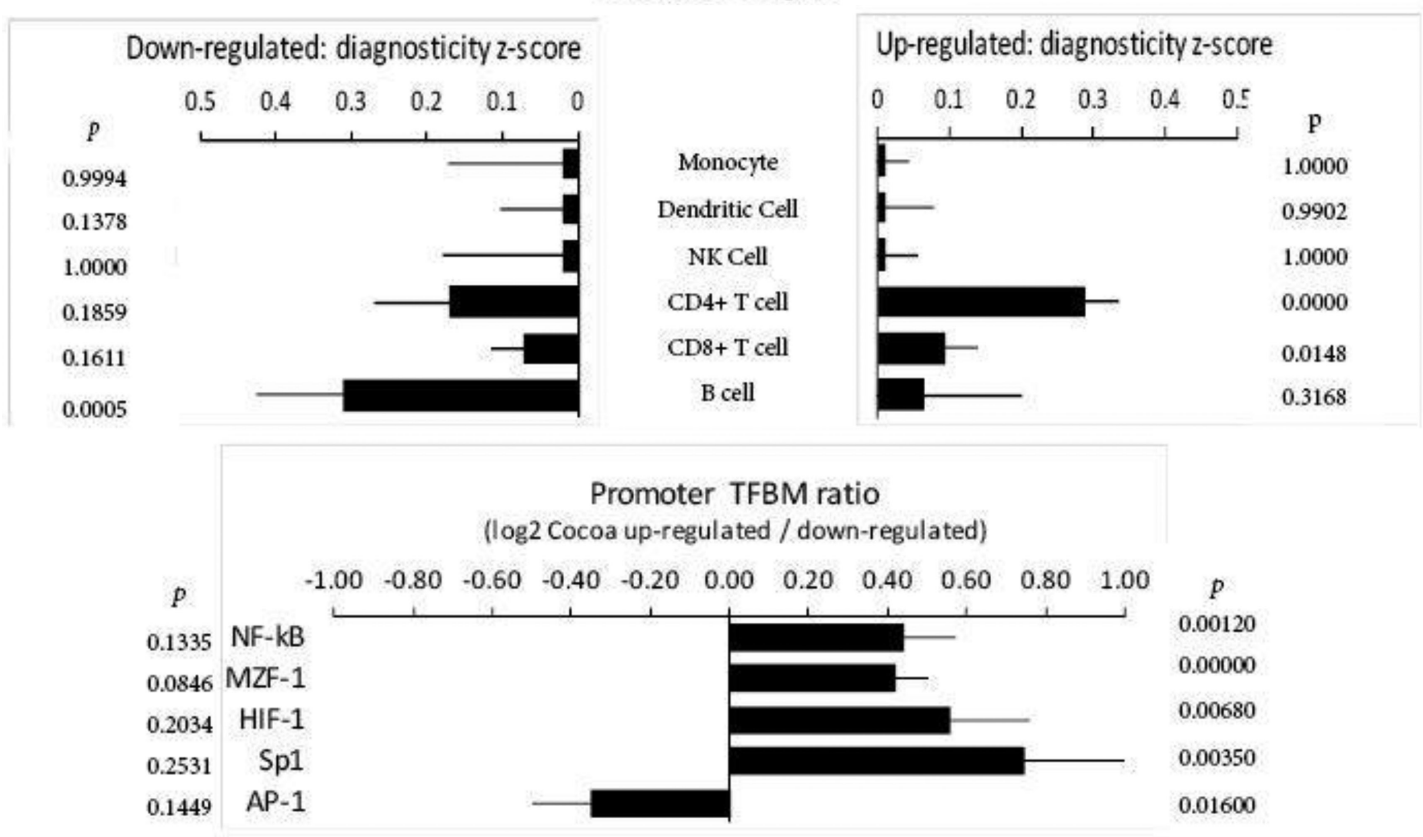

FIGURE P17-004-1 Modulation in transcription factor binding motifs for cacao administration/non-administration is indicated in a. with associated significance levels, b. Bioinformatically inferred for cellular origins of transcripts up- or down-regulated in differently expressed enes (e.g. up-regulated) are from the CD4+T cell population $(P=0.0)$ and (down-regulated) B cells $(P=0.0005)$. 


\section{Nutrients and DNA Methylation across the Life Course: A Systematic Review of Studies in Humans (P17-005)}

Kim VE Braun, Pooja Mandaviya, Oscar H Franco, Jana Nano, Carolin Girschik, Wichor Bramer, Taulant Muka, Jenna Troup, Joyce Meurs, Sandra Heil, and Trudy Voortman

Erasmus University Medical Center, The Netherlands

Objective: DNA methylation can be modified by environmental factors, including nutrition. In order to gain more insight into the effects of nutrients on DNA methylation, we conducted a systematic review on the relation between nutrients and DNA methylation in humans across the life course.

Methods: The literature search was designed by an experienced biomedical information specialist. Six bibliographic databases [Embase.com, Medline (Ovid), Web-of-Science, PubMed, Cochrane Central, and Google Scholar] were searched. We selected studies that examined the association between nutrients (blood levels, dietary intake, or dietary supplements) and DNA methylation (global, site specific, or genome wide) in humans of any age, with no restrictions on year of publication, language, or study design. Abstract screening, full-text selection, and data extraction were performed by 2 independent reviewers, with a third reviewer available to solve any disagreements.

Results: We identified 3774 references, of which 98 studies met all inclusion criteria. Most of these studies were performed in adult populations, and folate was the main nutrient of interest. Several candidate gene- and epigenome-wide association studies reported differential DNA methylation of $\mathrm{CpG}$ sites in response to folate (e.g., IGF2, H19, HOX), fatty acids (e.g., PPRAGC1A, TNF $\alpha$ ), and vitamin D (CYP24A1). Some of these observed associations were specific to life course stage (e.g., IGF2 in early life) and tissue (e.g., opposite directions for PPRAGC1A in muscle compared with fat tissue).

Conclusions: To date, promising results have been reported in the field of nutrition and DNA methylation in humans at different stages across the life course, especially for nutrients known to be involved in 1carbon metabolism, including folate, but also others, such as fatty acids and vitamin D. Studies on other nutrients, such as other macronutrients and various minerals, are still scarce. Further large-scale studies of high quality are needed to expand our understanding of the role of nutrition in DNA methylation and its effects on health and disease.

\section{Funding Sources}

The work presented in this abstract was conducted within ErasmusAGE at the Department of Epidemiology of Erasmus Medical Center, Rotterdam, The Netherlands. ErasmusAGE is a center for aging research across the life course and is funded by Nestlé Nutrition (Nestec Ltd) and Metagenics Inc.

Altered Expression Levels of Tet Methylcytosine Dioxygenases in Endothelial Progenitor Cells of Patients with Type 2 Diabetes with Complications (P17-006)

Guoxun Chen, ${ }^{1}$ Shi Zhao, ${ }^{2}$ Zihui Xu, ${ }^{2}$ Ting Jia, ${ }^{2}$ Yang Tang, ${ }^{3}$ Hong Mao, ${ }^{2}$ Wei Wei, ${ }^{2}$ Peirong Zhong, ${ }^{4} \mathrm{Lu} \mathrm{Ma},{ }^{4}$ and Rui $\mathrm{Li}^{4}$
${ }^{1}$ The University of Tennessee, Knoxville; ${ }^{2}$ Wuhan Central Hospital, China; ${ }^{3}$ Nanyang Technological University, China; and ${ }^{4}$ Wuhan University, China

Objective: Endothelial progenitor cells (EPCs) participate in microvascular repair, preventing complications in patients with diabetes. Epigenetic changes such as DNA methylation alter the functions of cells. Tet methylcytosine dioxygenases (TETs) are enzymes responsible for the conversion of 5-methylcytosine to 5-formylcytosine and 5-carboxylcytosine, cytosine derivatives of genomic DNA, during the process of demethylation. Here, we determined the levels of TET expression in EPCs of patients with diabetic complications.

Methods: Subjects who were nondiabetic (ND), diabetic only (D), and diabetic with complications (DC) were recruited for the collection of EPCs during June 2016-October 2016. EPCs from blood samples of $22 \mathrm{ND}, 29 \mathrm{D}$, and $22 \mathrm{DC}$ subjects, who provided signed informed consent forms, were isolated and subjected to analysis. The expression levels of TET1/2/3 mRNA and proteins were determined through the use of real-time polymerase chain reaction and immunoblot, respectively.

Results: The TET1 mRNA expression level in the ND group was higher than that in the D and DC groups. The TET3 mRNA level in the ND group was higher than that in the D group, which is higher than that in the DC group. The TET1 protein level in the DC group, but not in the $\mathrm{D}$ group, is higher than that in the ND group. The TET2 protein level in the DC group, but not in the D group, is lower than that in the ND group. The TET3 protein level in the ND group is higher than that in the D group, which is higher than that in the DC group. The TET3 protein level in the DC group is the lowest of the 3 groups. TET mRNA and protein levels largely matched each other.

Conclusions: The expression levels of TET1 and 3 proteins are lowered in EPCs of patients with diabetic complications, suggesting changes in genomic DNA methylation and the functions of EPCs. This phenomenon may contribute to microvascular complications in diabetic patients.

\section{Funding Sources}

The National Natural Science Foundation of China (grant 81370942 to SZ).

Candidate Gene and Genome-Wide Association Study for Umami Taste Perception: Genetic Risk Scores Including the TopRanked Polymorphisms and Association with Obesity Risk (P17007)

Oscar Coltell, ${ }^{1}$ Rebeca Fernandez-Carrion, ${ }^{2,3}$ Jose Sorli, ${ }^{2,3}$ Eva M Asensio, ${ }^{2,3}$ Rocio Barragan, ${ }^{2,3}$ Nadia San-Onofre, ${ }^{3}$ Carolina Ortega-Azorin, ${ }^{2,3}$ Paula Carrasco, ${ }^{3}$ Inmaculada GonzalezMonje, ${ }^{3}$ Esther Ferriz, ${ }^{3}$ and Dolores Corella ${ }^{2,3}$

${ }^{1}$ Universitat Jaume I-University of Valencia/CIBEROBN, Spain; ${ }^{2}$ CIBEROBN, Spain; and ${ }^{3}$ University of Valencia, Spain

Objective: Different taste perceptions may lead to distinctive food patterns that in turn may be related to obesity. Umami (pleasant savory), has been recognized as the "fifth taste." Some studies have associated umami substance with lower obesity risk. Some singlenucleotide polymorphisms (SNPs) in candidate genes, such as TAS1R1 
and TAS1R3 (taste receptors type 1 member 1 and member 3 ), have been associated with umami perception with heterogeneous results. Moreover, genome-wide association studies (GWASs) for umami taste are very scarce. We aimed at analyzing the association between SNPs ( $n=11$ in TAS1R 1 and $n=2$ in TAS1R3) in candidate umami genes as well as at the GWAS level for umami taste perception as well as the association between a genetic risk score (GRS) of the main SNPs and obesity risk.

Methods: In total, 356 participants in the PREDIMED PLUSValencia study were analyzed. Umami (monosodium L-glutamate, $200 \mathrm{mM}$ ) taste was determined through laboratory tests on the perception of their intensity (score 0-5). Selected candidate SNPs were: TAS1R1 (rs10864625, rs11122094, rs11122096, rs11122100, rs11587438, rs12041318, rs12565181, rs34857011, rs4908563, rs4908923, and rs4908932) and TAS1R3 (rs34810828 and rs35424002). Genotyping was undertaken with the Human OmniExpress Illumina array (700K) and GWAS was carried out with PLINK (additive model). Top-ranked SNPs $(P<0.00001)$ were identified and a weighted GRS with the independent SNPs $\left(r^{2}<0.2\right)$ was generated. Multivariate logistic regression was used for the association between GRS and obesity risk.

Results: None of the SNPs $(n=13)$ in the candidate genes was significantly associated with umami perception at $P<0.05$. We identified 14 GWAS-SNPs potentially associated with umami taste at $P<0.00001$. Nonrelated SNPs $(n=8)$ were included in the GRS for umami taste. The GRS was strongly associated with umami taste perception and obesity risk, decreasing the risk when increasing the number of umami taste perception alleles (OR: $0.79 ; 95 \% \mathrm{CI}: 0.65,0.97 ; P=0.022$, adjusted for age and sex).

Conclusions: We have detected new SNPs potentially associated with umami taste perception and a GRS of these SNPs is associated with decreased obesity risk.

Funding Sources

CIBEROBN (ISCIII, Madrid, Spain), PI16/00366 (ISCIII, Madrid Spain), PROMETEO17/2017 (Generalitat Valenciana, Valencia, Spain), University Jaume I, Castellon, Spain (P1-1B2013-54 and COGRUP/2016/06).

Gender Differences in the Perception of Salty Taste. Effect of the SCNN1B-rs239345 Polymorphism and its Use for Mendelian Randomization in a European Population (P17-008)

Dolores Corella, ${ }^{1,2}$ Olga Portoles, ${ }^{1}$ Jose Sorli, ${ }^{1,2}$ Jose González, ${ }^{1,2}$ Carolina Ortega, ${ }^{2}$ Oscar Coltell, ${ }^{3}$ Esther Ferriz, ${ }^{1}$ Eva M Asensio, ${ }^{1}$ and Rocio Barragan ${ }^{2}$

${ }^{1}$ University of Valencia, Spain; ${ }^{2}$ CIBEROBN, Spain; and ${ }^{3}$ Universitat Jaume I-University of Valencia/CIBEROBN, Spain

Objective: The aim of this study was to find out whether there is a difference in the perception of salty taste between men and women of a similar age in a general Mediterranean population, to analyze the influence of the rs239345 in Sodium Channel Epithelial 1 Beta Subunit (SCNN1B), a candidate gene for salty taste perception, and to assess whether this polymorphism would be a good proxy of salty taste for Mendelian randomized studies.
Methods: We analyzed 661 women and 368 men in a general Spanish population from the OBENUTIC study (mean \pm SD age $43 \pm 8 \mathrm{y}$ ). All were administered a test involving direct tasting of substances representing the different tastes (the intensity of perception was noted on a $0-5$ scale).

Results: Women perceived a significantly greater intensity of salty, sour, and bitter taste. There were no differences in sweet and umami. At $200 \mathrm{mM} \mathrm{NaCl}$, the mean of salty taste was $2.3 \pm 1.3$ points in men compared with $2.7 \pm 1.3$ in women $(P<0.000001)$. This difference remained statistically significant after adjusting for weight, diabetes, and lifestyle variables. The polymorphism was significantly and recessively associated with greater salty taste perception $(+0.35$ units for $200 \mathrm{mM}$ $\mathrm{NaCl}$ in homozygous subjects of the variant allele; $P=0.030$ ). No heterogeneity per gender was observed. Thus, this could be a good indicator of greater salty taste perception for use in Mendelian randomization. However, bearing in mind that many tastes are interrelated, we analyzed the association between the polymorphism and the perception of other tastes, and obtained a greater association with sweet taste perception (400 mM sucrose), +0.53 units in homozygous subjects $(P=0.001)$.

Conclusions: Women perceived more salty taste than men. Despite the rs239345 being significantly associated with greater salty taste perception, its great perception for sweet taste adds pleiotropy and limits its use as a proxy of salty taste in Mendelian randomization studies.

\section{Funding Sources}

CIBEROBN (ISCIII, Madrid, Spain), PROMETEO17/2017 (Generalitat Valenciana, Valencia, Spain).

Gene-Based Analysis of Angiogenesis-Related Pathways in Skeletal Muscle of Older Individuals Following Cocoa Flavanol or Fish Oil Supplementation (P17-009)

Hannah Crossland, ${ }^{1}$ Suzette Pereira, ${ }^{2}$ Kenneth Smith, ${ }^{1}$ Bethan Phillips, ${ }^{1}$ and Philip Atherton ${ }^{1}$

${ }^{1}$ University of Nottingham, United Kingdom; and ${ }^{2}$ Abbott Nutrition, $\mathrm{OH}$

Objective: Aging is associated with impaired muscle microvascular function and extracellular matrix (ECM) remodeling, insulin sensitivity, and mitochondrial abundance/function. Cocoa flavanols and fish oil omega-3 (n-3) fatty acids have emerged as two bioactive nutrients that may affect these properties, but the mechanisms by which they mediate these effects remain unclear. We evaluated changes in gene expression of these pathways in muscle from nutritional intervention studies in older adults.

Methods: Real-time polymerase chain reaction was carried out on muscle from 3 studies ( $n=8$ /group): 1 ) pre- and acute post-feed cocoa flavanols in older males [350 mg; mean \pm SD age: $72.6 \pm$ $1.6 \mathrm{y}$; body mass index (BMI, $\left.\left.\mathrm{kg} / \mathrm{m}^{2}\right): 26.7 \pm 0.6\right]$; 2) post- 1 wk daily cocoa flavanol supplementation in older males compared with noncocoa control group (350 mg/d; age: $70.1 \pm 0.9$ y; BMI: $25.7 \pm 0.6$ ); and 3) pre-/post-2 and $6 \mathrm{wk}$ fish oil supplementation in older females (3.4 g/d; age: $64.4 \pm 0.8 \mathrm{y}$; BMI: $26.2 \pm 0.7$ ). Genes were subcategorized based on ontology, and 2-way ANOVA was used to assess main effects of 
treatment. Sidak's tests determined changes in gene expression between groups.

Results: There was a main effect of 6-wk fish oil on angiogenesis gene expression, with increases in ANGPTL4, EPHB2, and CXCL10 (all $P<0.05$ compared with week 0 ). Analysis of mitochondrial and insulin signaling genes with 2 or $6 \mathrm{wk}$ of fish oil treatment showed no overall changes; however, there was an increase in MTOR at $2 \mathrm{wk}$, and in PDK4 and IGF1 at $6 \mathrm{wk}(P<0.05$ compared with week 0$)$. Acute cocoa caused an overall change in angiogenesis genes, with significant increases in THBS1, ADAMTS1, and CXCL2 $(P<0.05$ compared with pre-cocoa). Finally, although 1 wk of cocoa did not result in an overall change in angiogenesis gene expression, there were increases in $C T G F$ and FGF1 expression $(P<0.05$ compared with non-cocoa). Followup analysis showed significant increases in COL1A1, COL1A2, and COL3A1 $(P<0.05$ compared with non-cocoa) with cocoa treatment.

Conclusions: There is evidence of changes in angiogenesis gene expression following $6 \mathrm{wk}$ of fish oil supplementation, as well as increased anabolism-related genes, consistent with previous observations. Acute and daily cocoa supplementation for $1 \mathrm{wk}$ were associated with changes in angiogenesis genes, with ECM remodeling emerging as an area for further study.

\section{Funding Sources}

Abbott Nutrition.

Essential Trace Elements and Promoter DNA Methylation of Mineral Transporter Genes: Unravelling Novel Epigenetic Mechanisms in Human Hepatocarcinoma (P17-010)

Domenica De Santis, ${ }^{1}$ Silvia Udali, ${ }^{1}$ Filippo Mazzi, ${ }^{1}$ Andrea Ruzzenente, ${ }^{1}$ Greta Beschin, ${ }^{1}$ Patrizia Pattini, ${ }^{1}$ Antonia Franceschi, ${ }^{1}$ Sara Moruzzi, ${ }^{1}$ Luigi Perbellini, ${ }^{1}$ Alfredo Guglielmi, ${ }^{1}$ Oliviero Olivieri, ${ }^{1}$ Sang Woon Choi ${ }^{2,3}$ and Simonetta Friso ${ }^{1}$

1 University of Verona, Italy; and ${ }^{2}$ Chaum Life Center, CHA University, South Korea; and ${ }^{3}$ Tufts University School of Nutrition Science and Policy, MA

Objectives: Hepatocellular carcinoma (HCC) is the second most common cause of death from cancer worldwide. The etiology and factors involved in its development remain, however, largely unknown. A marked decrease in mineral trace elements is related to the genesis of several diseases including cancer but their role in carcinogenesis, including that of HCC, is yet not fully defined. We investigated an ample set of trace elements amounts in serum, HCC tissues, and homologous non-neoplastic liver tissues (N) from HCC patients. Gene expression and promoter DNA methylation of major element-transport proteins were also determined. We aimed to study the possible involvement of DNA methylation in trace element-linked protein dysregulation and eventually in HCC progression.

Methods: Copper, zinc, selenium, cadmium, and cobalt were measured in tissues and serum of HCC patients undergoing hepatic curative surgery by inductively coupled plasma mass spectrometry analysis. Gene expression profiles and DNA methylation at promoter of zinc-transporter (ZIP8, ZIP14) and metallothionein (MT1G, MT1H, $M T 2)$ genes were determined by a microarray genome-wide approach.
Results: Serum concentrations of copper, zinc, and selenium did not show differences and were within the reference limits among all the patients. Tissue concentrations of zinc, cadmium, selenium, and cobalt were, instead, markedly decreased in HCC as compared with normal tissues; copper concentrations did not differ significantly between HCC and $\mathrm{N}$ tissues. Moreover, ZIP8, ZIP14, MT1G, MT1H, and MT2 genes were repressed with consensual promoter hypermethylation in HCC liver tissues as compared with $\mathrm{N}$ tissues.

Conclusions: We show the novel finding of a significant reduction of zinc and cadmium concentrations in HCC tissues, together with a gene repression of the metal-transporters ZIP8, ZIP14, MT1, and MT2 linked to promoter gene hypermethylation, suggesting an early, epigeneticbased dysregulation of zinc and cadmium depletion in HCC. These findings provide new insights into the role of trace elements in HCC carcinogenesis.

Characterizing the Genetics of Salt Sensitivity: A Cost-Efficient Assay in Development (P17-011)

Olivia Dong, ${ }^{1}$ Oscar Suzuki, ${ }^{1}$ Rachel M Howard, ${ }^{1}$ Kelly Jamieson, ${ }^{1}$ Mildred Pointer, ${ }^{2}$ Martin Kohlmeier, ${ }^{3}$ and Tim Wiltshire $^{1}$

${ }^{1}$ University of North Carolina at Chapel Hill; ${ }^{2}$ North Carolina Central University; and ${ }^{3}$ University of North Carolina Nutrition Research Institute

Objective: The aim of this study was to develop a cost-efficient genetic assay that interrogates extensive genomic regions to identify genetic markers associated with the salt-sensitivity (SS) phenotype. The ultimate goal of developing this genetic assay is for future use in cohorts where the SS phenotype has been determined to distinguish responders to low-sodium intake from nonresponders.

Methods: A genetic assay was developed to sequence $\sim 124 \mathrm{~kb}$ of the genome consisting of 33 genes that have been linked to SS at various levels of evidence (i.e., highly correlated or inconclusively correlated). All coding exons and promoter regions for 13 genes and single-nucleotide polymorphisms (SNPs) in 20 genes associated with SS are captured on the assay. The assay utilizes a custom-capture method that uses molecular inversion probes (MIPs) to generate a library of DNA fragments for next-generation sequencing (NGS) on the Illumina MiSeq platform. Each molecular inversion probe captures 112 nucleotides of the genome and 1089 MIPs are combined to capture relevant genetic information in targeted genomic regions associated with SS. A minimum sequencing depth of $20 \times$ is required for genotyping and haplotype calling, and the concentration of each MIP is optimized to achieve this coverage threshold. The cost of processing one sample includes DNA extraction, library preparation and NGS, and personnel time.

Results: A MiSeq run multiplexing 62 DNA samples from the 1000 Genomes Project was completed through the use of the genetic assay. Average coverage across all genes for the 62 samples was $200 \times$ (range: $8 \times-663 \times)$. Four genes had an average of $<20 \times$ average coverage. The cost of processing one sample was $\$ 63$ or $\$ 1.91$ per gene. This cost includes DNA extraction (\$1), library preparation and NGS costs (\$35), and personnel time (\$17). 
Conclusions: The results suggest that customized MIPs for NGS can efficiently interrogate extensive genomic regions related to SS. The modest cost makes sequencing for large numbers of people practical and affordable. This low-cost genetic assay holds promise as a practical tool for predicting adverse response to dietary sodium intake and helping health care providers to tailor intervention efforts more effectively to salt-sensitive patients.

\section{Funding Sources}

The UNC Center for Pharmacogenomics and Individualized Therapy and North Carolina Central University provided funding for this project.

Expression of the Hypocretin-1 gene (Orexin-A) in Severely Obese Women Submitted to Roux-en-Y Gastric Bypass (P17-012)

Rayana Foglietti, Barbara dos Santos, Caroline Welendorf, Flavia C Ferreira, Leticia Wolf, Carolina Nicoletti, Marcela Augusta Pinhel, Bruno Parenti, Vitor C Pinhanelli, and Carla B Nonino

University of São Paulo, Brazil

Objective: The aim of this study was to verify the expression of the gene for Orexin-A in women with severe obesity submitted to Roux-en$\mathrm{Y}$ gastric bypass.

Methods: Patients were selected in a university hospital and distributed into 2 groups: 1$)$ the bariatric group $(n=10)$ was composed of women with severe obesity [body mass index (BMI; $\left.\mathrm{kg} / \mathrm{m}^{2}\right)>35$ ] submitted to surgery; and 2) the control group $(n=7)$ was composed of women with a BMI between 18.5 and 24.9. Before and after the surgical procedure, anthropometric data (weight, height, BMI, and waist circumference), body composition, food intake (24-h food recall), and peripheral blood collection were analyzed for Orexin-A gene expression.

Results: Orexin-A gene expression was increased 6 mo after bariatric surgery (mean $\pm \mathrm{SD}$ : $5.38 \pm 0.1$ ) compared with the preoperative period $(-1.64 \pm 0.08 ; P>0.05)$. Significant reduction was observed in all anthropometric and body composition indicators $(P<0.05)$. Regarding the indicators of food intake, there were no significant changes.

Conclusion: Significant weight loss within 6 mo after bariatric surgery promotes a considerable increase in Orexin-A gene expression. In addition, it positively aids changes in anthropometric parameters and body composition.

\section{Funding Sources}

Fundação de Amparo à Pesquisa do Estado de São Paulo (FAPESP), Fundação de Apoio ao Ensino, Pesquisa e Assistência (FAEPA).

Effects of Resistant Starch on Selecting Housekeeping Genes and Gluconeogenesis Regulatory Enzyme (P17-013)

Justin Guice, ${ }^{1}$ Zach Bendiks, ${ }^{2}$ David Welsh, ${ }^{3}$ Meng Luo, ${ }^{3}$ Chris M Taylor, ${ }^{3}$ Eugene Blanchard, ${ }^{3}$ Anne M Raggio, ${ }^{1}$ Claudia Husseneder, ${ }^{1}$ Ryan Page, ${ }^{1}$ Brian D Marx, ${ }^{4}$ Diana Coulon, ${ }^{1}$ Maria Marco, ${ }^{2}$ and Michael J Keenan ${ }^{1}$

${ }^{1}$ Louisiana State University AgCenter; ${ }^{2}$ University of California, Davis; ${ }^{3}$ Louisiana State University Health Sciences Center; and ${ }^{4}$ Louisiana State University

Objectives: Gene expression analyses require validated housekeeping genes (HKGs) that maintain uniform expression under controlled and experimental conditions. Our objectives were to assess the effect of resistant starch (RS) fermentation in rats on commonly used HKGs and also of the gluconeogenesis regulatory enzymes essential for regulating glucose homeostasis in the cecum, specifically phosphoenolpyruvate carboxykinase (PEPCK1). Currently, there is no universal consensus on the occurrence of intestinal gluconeogenesis (IGN).

Methods: In a $2 \times 2 \times 2$ factorial arrangement, 8 dietary treatments were fed to Sprague Dawley (SD) rats for $6 \mathrm{wk}$. Treatment factors were RS (present/absent), whole grain (present/absent), and fat (high, moderate). Through the use of quantitative reverse transcriptasepolymerase chain reaction (RT-qPCR), a commonly used HKG, cyclophilin F (PPIF), was tested to determine any statistically significant effect of dietary RS treatment. Gene expression (RT-qPCR) and protein produced (Western blot and ELISA) were determined for PEPCK1 in the cecum. In a second study $(2 \times 2$ factorial $), 4$ dietary treatments were fed to SD rats for $12 \mathrm{wk}$. Treatment factors were: sodium butyrate (SB) and RS (present/absent). To compare effects of possible dietary RS treatment effect on HKG expression with the first study, we then performed RT-qPCR and statistical analysis on $18 \mathrm{~S}$ rRNA and PPIF genes.

Results: In both studies, dietary RS fermentation significantly increased PPIF gene expression $(P \leq 0.002)$, whereas all other dietary treatments were not significantly different. In study 2 , the $18 \mathrm{~S}$ rRNA gene was not significantly different $(P \geq 0.18)$ for dietary RS. Western blot produced no band for PEPCK1, whereas ELISA was effective for protein quantitation. A significant increase in PEPCK1 gene expression $(P<0.0001)$ and protein $(P \leq 0.001)$ produced was observed when dietary RS was present.

Conclusions: Increased PPIF gene expression, when used as a HKG, biases against an RS effect. However, despite PPIF bias, significance is still attained with dietary RS due to robust effects of fermentation, even in one rodent study with RS as low as $5 \%(\mathrm{w} / \mathrm{w})$. We recommend that the 18S rRNA gene be used as the HKG to avoid overlooking changes that are subtle in comparison to the robust RS fermentation. Dietary RS positively affects gene expression and protein production of PEPCK1, illustrating support for the cecum as a site for IGN.

\section{Funding Sources}

USDA-NIFA \#2014-67017-21760, LSU AgCenter. Ingredion Incorporated gifted flour/starches.

Polymorphisms in the Reverse Cholesterol Transport Pathway Cumulatively Affect the Amounts of Triglycerides in Young Mexican Adults (P17-014)

Bridget A Hannon, ${ }^{1}$ Itzel Vasquez-Vidal, ${ }^{2}$ Flavia Andrade, ${ }^{1}$ Celia Aradillas-Garcia, ${ }^{3}$ Naiman Khan, ${ }^{1}$ and Margarita Teran-Garcia ${ }^{1}$

${ }^{1}$ University of Illinois at Urbana-Champaign; ${ }^{2}$ University of Manitoba, Canada; and ${ }^{3}$ Autonomous University of San Luis Potosi, Mexico

Objective: ATP-binding cassette 1 (ABCA1) and cholesterol-ester transfer protein (CETP) are proteins that participate in the reverse cholesterol transport (RCT) pathway, which facilitates cholesterol return to the liver via high-density lipoprotein (HDL) cholesterol particles. Genetic variants, in the form of single-nucleotide polymorphisms (SNPs), may affect the efficiency of this process, resulting in low HDL and high triglyceride (TG) concentrations. Diet also influences these biomarkers, specifically carbohydrates and saturated 
fat (SFA). Nutrigenetic interactions between diet and variants in RCT may contribute to dyslipidemia. We aim to determine how cumulative risk of multiple SNPs in this pathway relates to diet and the amounts of lipoproteins.

Methods: Subjects were Mexican young adults (aged 18-25 y; $n=649 ; 53 \%$ female) from the UPAMIGOS cohort study. Fasting blood was collected for genetic and lipoprotein analysis. Dietary intake was also collected, and carbohydrates and SFAs were expressed as percentage of total calories. SNPs from ABCA1 (rs9282541 and rs4149310) and CETP (rs1532624, rs289714, and rs5882) were selected based on their classification as functional variants. Genotypes were assigned 0,1 , or 2 , corresponding to the number of risk alleles for each SNP. Cumulative risk was calculated from the sum of all alleles. Generalized linear models were adjusted for age, sex, and body mass index.

Results: SNPs were not in linkage disequilibrium, and had a minor allele frequency $>10 \%$. Cumulative genetic risk was associated with higher TG $\left(P=0.001, r^{2}=0.20\right)$. The mean number of risk alleles was 3.9. Individuals with $\geq 4$ risk alleles had greater concentrations of TG compared with those with lower risk (115.1 compared with $105.6 \mathrm{mg} / \mathrm{dL} ; P=0.001)$. SFA intake was not found to be significantly predictive of TG concentrations. However, carbohydrate intake was associated with high TG concentrations in the cumulative risk model $(\beta=0.19, P=0.025)$

Conclusions: Common variants involved in RCT cumulatively influence TG concentrations in young adults. TG concentrations were also affected by carbohydrate, but not SFA intake. Carbohydrates promote high TG concentrations by increasing hepatic lipogenesis, and multiple variants in RCT may result in accumulation of TG-rich lipoproteins. This study provides novel information on the aggregate effects of pathway-specific variants and diet on TG in an understudied population.

\section{Funding Sources}

This study was funded by University of Illinois at UrbanaChampaign Research Board grant 09070 (to FA), the Center for Health and Aging (to FA), and Hatch Projects ILLU-968-312, ILLU-971-368, and ILLU-793-327 (to MT-G). Funding was also provided by the Universidad Autónoma de San Luis Potosí, Hormones Laboratory at the School of Medicine, the Clinical Biochemistry Laboratory at the Chemical Sciences School, and the Autonomous University of San Luis Potosí University Health Center under agreement support C09-PIFI030606 (to CA-G). BAH was supported by the National Institute for Agriculture under the AFRI Childhood Obesity Prevention Challenge Area grant (2015-68001-23248) to the University of Illinois.

Genome-Wide Analysis of DNA Methylation in an Intervention Study of the Traditional Korean Diet (P17-015)

Moonju Hong, ${ }^{1}$ Jae-jo Park, ${ }^{1}$ Hee-Sook Park, ${ }^{1}$ Soon-Hee Kim, ${ }^{1}$ Sang-Woon Choi, ${ }^{2}$ Hae-Jeung Lee, ${ }^{3}$ Seon-Joo Park, ${ }^{3}$ Phil-Kyung Shin, ${ }^{2}$ and Myung-Sunny Kim ${ }^{1}$

${ }^{1}$ Korea Food Research Institute in University of Science and Technology; ${ }^{2}$ CHA University, South Korea; and ${ }^{3}$ Gachon University, South Korea

Objective: The objective of the study was to understand the relation between metabolic diseases and epigenetic changes due to diet by investigating changes in DNA methylation in premenopausal women consuming a traditional Korean diet (K-diet).

Methods: We recruited 10 overweight perimenopausal women, and randomly divided these into 2 groups: 1$)$ the K-diet group $(n=5)$, who consumed multigrain rice, various fermented foods, vegetables, legumes, fish, and low red meat; and 2) the control diet group $(n=5)$, who consumed a Westernized Korean diet. All subjects stayed at the same metabolic unit for $2 \mathrm{wk}$ of dietary intervention. Clinical indicators including total blood cholesterol, fibrinogen, insulin, and platelet were measured as instructed in blood collected at baseline, $1 \mathrm{wk}$, and $2 \mathrm{wk}$. Whole-genome bisulfite sequencing was performed in blood cell DNA. Trimmed reads were aligned and methylation determined through the use of BS-seeker2 with Bowtie2. Differentially methylated regions (DMRs) between groups were analyzed within 100 bp with a 50-bp step size window and annotated by HOMER. Spearman correlation was analyzed with the $\mathrm{R}$ package corrplot. Bisulfite amplicon sequencing was used to validate the methylated region.

Results: Two weeks of K-diet significantly reduced blood total cholesterol concentrations $(239.40 \pm 15.14$ compared with $198.20 \pm 13.25 \mathrm{mg} / \mathrm{dL}, P=0.0163$ ), but control diet had no such effect. A significant interaction between time and diet group was observed in the total blood cholesterol $(P$-interaction $=0.0034)$. A total of 85,976 DMRs (K-diet) and 87,621 DMRs (control diet) were significantly differential methylated. $(P<0.05$, methylation differences $>5 \%)$. We identified 13 DMRs where significantly differential methylation between K-diet and control diet was proved by bisulfite amplicon sequencing. Among 13 DMRs, a Spearman correlation test revealed that there were statistically significant correlations between 8 DMRs and clinical indicators, and between 5 DMRs and clinical dietary compositions (11 food groups, 44 nutrients).

Conclusions: The findings indicate that DNA methylation in blood cells was significantly regulated by dietary pattern. We suggest that the traditional Korean diet can modulate metabolic disease-associated genes that are highly correlated with clinical indicators and dietary compositions through modification of DNA methylation.

\section{Funding Sources}

Korea Food Research Institute.

Vitamin $D_{3}$ and Sulforaphane Effects on RON Receptor Tyrosine Kinase in Human Colorectal (Caco2) and Breast Cancer (MCF7) Cells (P17-016)

Sharmin Hossain, Zhenhua Liu, and Richard Wood

University of Massachusetts Amherst

Objective: The RON receptor tyrosine kinase is overexpressed in $\sim 50 \%$ of human breast tumors, and is significantly increased in most primary human colorectal adenocarcinomas. 1,25Dihydroxycholecalciferol $\left[1,25(\mathrm{OH})_{2}-\mathrm{D}_{3}\right.$, vitamin $\left.\mathrm{D}_{3}\right]$ protects against mammary tumor formation, progression, and metastasis, whereas sulforaphane (SFN) exerts its action via histone deacetylase (HDAC) inhibition in cancer epigenetics. RON overexpression in mammary tumors is a result of accelerated tumor cell proliferation, migration, and invasion. We hypothesized that vitamin $\mathrm{D}_{3}$ and SFN will have a protective role in $\mathrm{RON}$-induced tumorigenesis in colorectal and breast cancer. The objective of the present study is to decrease RON expression upon treatment with vitamin $\mathrm{D}_{3}$ and SFN (alone and in combination) in human colorectal and breast cancer cells. 
Methods: We treated Caco-2 (colorectal cancer) and MCF-7 (breast cancer) cells with $100 \mathrm{nM}$ vitamin $\mathrm{D}_{3}, 20 \mu \mathrm{M}$ SFN, and $1 \mu \mathrm{M}$ trichostatin A (TSA, a histone deacetylase inhibitor used as a positive control) for $24 \mathrm{~h}$ post- $70 \%$ confluency. We repeated the experiment in fully differentiated Caco- 2 cells as well. The effects of SFN and TSA with and without the presence of vitamin D were tested on Caco-2 and MCF7 cells.

Results: RON expression was significantly decreased in SFN alone $\left(\mathrm{pR}^{2}=0.98\right)$; $\mathrm{D}$ alone $(\mathrm{p} 2=0.90)$ as Caco-2 except the $\mathrm{D}+$ TSA group $(P=0.29,-0.31 \pm 0.16) ; \mathrm{D}$ alone $(\mathrm{p}$

Conclusions: Vitamin $D_{3}$ alone and vitamin $D_{3}$ in combination with SFN have strong nutrigenomic intervention potentials in colorectal and breast cancer through inhibition of histone deacetylation and subsequent decrease in RON-dependent cell-cycle events.

\section{Funding Sources}

This research was funded by the United States Department of Agriculture (USDA) Hatch Grant MAS 00992.

\section{Supporting Images/Graphs}

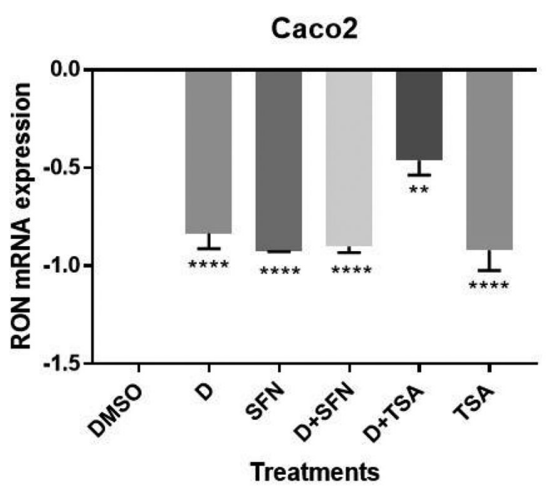

FIGURE P17-016-1 RON mRNA expression in differentiated Caco2 cells

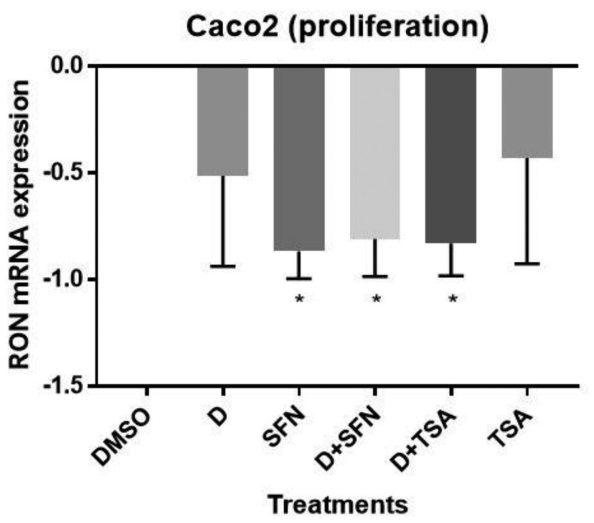

FIGURE P17-016-2 RON mRNA expression in proliferating Caco2 cells

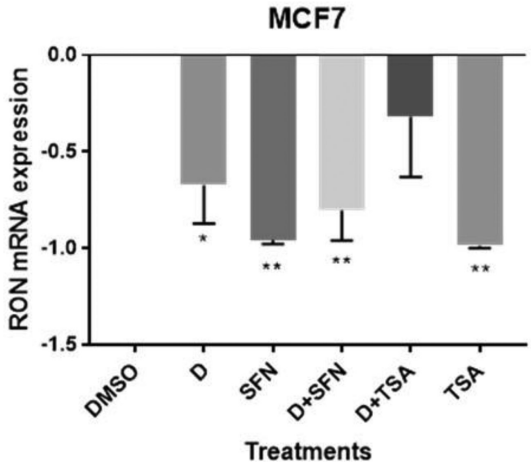

FIGURE P17-016-3 RON mRNA expression in MCF7 cells

The Influence of the Gut Microbiota on Response to Diet: A Randomized, Controlled Crossover Study of Resistant Starch Wheat (P17-017)

Riley L Hughes, ${ }^{1}$ Nancy Keim, ${ }^{2}$ Mary Kable, ${ }^{2}$ and Danielle Lemay $^{2}$

${ }^{1}$ University of California, Davis; and ${ }^{2}$ Western Human Nutrition Research Center (USDA-Agricultural Research Service)

Objective: This study aims to examine some of the interindividual variation in human response to a dietary intervention by identifying features of the microbiome associated with this variability and making use of these features to develop a model to predict metabolic [i.e., glycemic response, small-chain fatty acids (SCFAs), bile acids, breath hydrogen/methane] and microbiota response to dietary fiber in an independent cohort.

Methods: Metabolic responses (e.g., glycemic response, SCFA production) and $16 \mathrm{~S}$ rRNA gene data being generated from a doubleblind, placebo-controlled, crossover clinical trial of resistant starch (RS) will be used to investigate whether baseline features of the microbiota are correlated with interindividual differences in the effect of RS supplementation in individuals. Linear mixed models of glycemic response and features of the gut microbiota hypothesized to modify the effect of the intervention as well as covariates (e.g., gender, habitual fiber intake) will be used to determine microbiota features associated with improvement in glycemic response as a result of RS supplementation.

Results: Preliminary analysis of data from the 11 participants who have completed the study to date shows an overall nonsignificant effect on fermentation, as indicated by breath hydrogen $\left(\mathrm{H}_{2}, P=0.110\right)$ and methane $\left(\mathrm{CH}_{4}, P=0.101\right)$. However, interindividual variability in the direction and magnitude of the response in breath $\mathrm{H}_{2}$ and $\mathrm{CH}_{4}$ to RS compared with placebo treatments indicates varying degrees of efficacy of RS to stimulate fermentation by the individual's resident gut microbiota.

Conclusions: The results of this study indicate interindividual variability in response to fiber (RS) supplementation. Determination of microbiota features that associate with this variability will contribute to our ability to predict and potentially modify an individual's response to diet.

Funding Sources

Innovation Institute for Food and Health (IIFH) at UC Davis. 

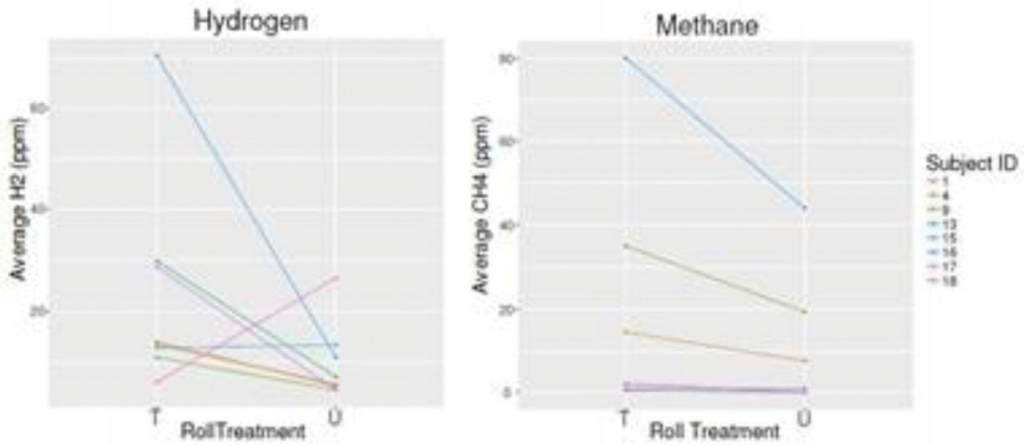

FIGURE P17-017-1 Inter-individual variation in breath hydrogen and methone response to roll treatment Roll treatments remain blinded

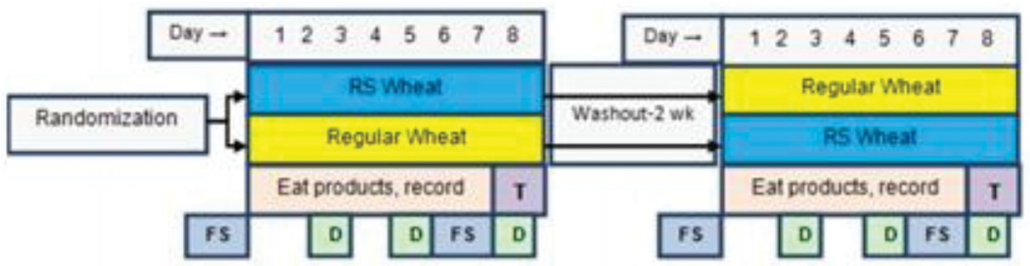

FIGURE P17-017-2 Study design and timeline. T, meal challenge test day; D, dietary recall; FS, fecal sample collection window.

The Associations of Genetic Variants at Apolipoprotein C3 with Incident Hypertension Are Modified by Obesity and Physical Activity in Korean Adults (P17-018)

Garam Jo, So-Young Kwak, and Min-Jeong Shin

Korea University

Objectives: We investigated whether triglyceride (TG)-influencing genetic variants at Apolipoprotein C3 (APOC3) are associated with the development of hypertension (HTN), and whether these associations are modified by lifestyle measures in Koreans.

Methods: Data were obtained from a subset of the AnsungAnsan cohort within the Korean Genome and Epidemiology study (KoGES-ASAS). After excluding participants with pre-existing cancer, cardiovascular diseases, diabetes, HTN, and TG concentrations $>600$ $\mathrm{mg} / \mathrm{dL}$, a total of 5239 Koreans were included at baseline (2011-2012), and followed up for $9.8 \mathrm{y}$. Low physical activity was defined by metabolic equivalents (hours per day) lower than median in total subjects.

Results: The minor allele frequencies of APOC3 gene polymorphisms (rs2854117, rs4520, and rs5128) in the KoGES-ASAS groups were $0.464,0.403$, and 0.324 for all subjects, respectively. The results showed that the subjects with minor alleles in rs2854117 and rs5128 were significantly associated with higher circulating TG concentrations $(P=0.006)$, whereas the subjects with minor alleles of rs4520 showed significantly lower circulating TG concentrations $(P<0.001)$ and higher high-density lipoprotein cholesterol concentrations $(P=0.011)$. Although no association was found between genetic variants at APOC3 and the risk for HTN, the genotype effects on HTN were found to be modified by obesity and regular physical activity. Specifically, the minor C allele of rs4520 showed a significantly lower risk for HTN (HR: 0.87; $95 \%$ CI: $0.78,0.97)$ only in normal-weight subjects [body mass index $\left(\mathrm{kg} / \mathrm{m}^{2}\right)<25$ ] after adjusting for covariates. In addition, the T allele of rs 2854117 was positively related to the incident HTN only in the subjects characterized by low physical activity (HR: 1.13; 95\% CI: 1.02, 1.26).

Conclusion: Our results suggest that the genotype effects of APOC 3 on the risk of HTN were modified by obesity and physical activity in a large Korean population.

\section{Funding Sources}

This study was provided with bioresources from the National Biobank of Korea, the Centers for Disease Control and Prevention, Republic of Korea (KBP-2016-062). This research was supported by the Basic Science Research Program through the National Research Foundation of Korea (NRF), which is funded by the Ministry of Education, Science, and Technology (NRF-2015R1A2A1A15054758).

Lack of Causal Association between Circulating 25Hydroxyvitamin D Concentrations and Hypertension in Korean Adults: A 2-Sample Mendelian Randomization Study (P17-019)

So-Young Kwak, ${ }^{1}$ Garam Jo, ${ }^{1}$ Yoonsu Cho, ${ }^{2}$ and Min-Jeong Shin ${ }^{1}$

${ }^{1}$ Korea University; and ${ }^{2}$ University of Bristol, United Kingdom

Objective: Low circulating 25-hydroxyvitamin D [25(OH)- $\left.\mathrm{D}_{3}\right]$ concentrations have been associated with a higher risk for hypertension 
(HTN). However, their causal relationship is still unclear. Mendelian randomization (MR) is an approach that can investigate causal effects of risk factor on outcomes based on the use of genetic variant as an instrumental variable. In this study, we aimed to evaluate a causal relationship between $25(\mathrm{OH})-\mathrm{D}_{3}$ concentrations and $\mathrm{HTN}$ in a Korean population through the use of a 2 -sample MR approach.

Methods: We examined the causal effect of $25(\mathrm{OH})-\mathrm{D}_{3}$ on the prevalence of HTN from 2 nonoverlapping samples. 25(OH)- $\mathrm{D}_{3}$ related single-nucleotide polymorphisms (SNPs; DHCR7 rs12785878, CYP2R1 rs10741657 and rs12794714, and GC rs2282679) identified from genome-wide association studies were chosen as genotypic proxies for circulating $25(\mathrm{OH})-\mathrm{D}_{3}$ concentrations. The association between SNP and $25(\mathrm{OH})-\mathrm{D}_{3}$ was estimated from 2595 subjects from a subset of the Korean National Health and Nutrition Survey 2011-2012 (sample 1); and the association between SNP and HTN was estimated from 8343 subjects from the Korean Genome and Epidemiology Study (sample 2). Three different analytic methods were carried out for the main analysis and the sensitivity analysis of the 2-sample MR: inverse variance weighted (IVW), MR-Egger regression, and weighted median regression.

Results: Circulating $25(\mathrm{OH})-\mathrm{D}_{3}$ concentrations were inversely associated with the prevalence of HTN in ordinary least-squares analysis (OR $=0.97$; 95\% CI: 0.96, 0.99) after adjusting for potential covariates. Main analysis performed with IVW indicated that there was no causal association between $25(\mathrm{OH})-\mathrm{D}_{3}$ concentrations based on genotypic proxies with prevalence of HTN (OR $=1.01 ; 95 \%$ CI: 0.97, 1.05). For sensitivity analysis, both the MR-Egger and weighted median methods reported no causal association between $25(\mathrm{OH})-\mathrm{D}_{3}$ concentrations and prevalence of $\mathrm{HTN}(\mathrm{OR}=0.96 ; 95 \%$ CI: $0.78,1.17$ for MR-Egger; OR $=1.01 ; 95 \%$ CI: $0.96,1.06$ for weighted median method). Hence the results from using IVW and two pleiotropy-adjusted analyses (MR-Egger and weighted median method) showed consistently that the causal effects were found to be null.

Conclusions: This MR study found no evidence for circulating $25(\mathrm{OH})-\mathrm{D}_{3}$ concentrations as a causal risk factor for the prevalence of HTN in Korean adults.

\section{Funding Sources}

This study was provided with bioresources from the National Biobank of Korea, the Centers for Disease Control and Prevention, Republic of Korea (KBP-2016-009). This research was supported by the Basic Science Research Program through the National Research Foundation of Korea (NRF) funded by the Ministry of Education, Science and Technology (NRF-2015R1A2A1A15054758).

The Association between Genetic Variants at Angiopoietin-Like 3 and Diabetes Risk in Korean Population: Results from the Korean Genome and Epidemiology Study (P17-020)

Juhee Lee, So-Young Kwak, Garam Jo, Ji Young Kim, and MinJeong Shin

\section{Korea University}

Objective: Angiopoietin-like 3 (ANGPTL3) has emerged as an important regulator of circulating triglyceride (TG) and cholesterol concentrations through inhibition of lipoprotein lipase (LPL) and endothelial lipase enzyme activity. Several previous studies have reported that common genetic variants at ANGPTL3 were associated with lower circulating concentrations of TGs and total cholesterol (TC) in the general population. In this study, we tested possible protective effects against the development of type 2 diabetes (T2D) based on the previously reported association between genetic variations of ANGPTL3 and lifelong reduced concentrations of TC and TGs.

Methods: A prospective cohort study was designed to evaluate the association of genetic variants at ANGPTL3 gene (rs11207997, rs10789117, and rs17123721) with the incidence of T2D. We analyzed a total of 7384 adults (3436 men and 3948 women, aged 40-69 y) without cancer, cardiovascular disease, and T2D at baseline from the Ansan-Ansung cohort of the Korean Genome and Epidemiology Study (KoGES). Participants had 5 follow-up examinations over $10 \mathrm{y}$. Linear regression analysis was used to test the association between ANGPTL3 genetic polymorphisms and various clinical parameters, and the Cox proportional hazard regression model was used to evaluate the HR with 95\% CI, adjusting for potential covariates.

Results: Linear regression analyses showed that genetic variants at ANGPTL3 (rs11207997 and rs10789117) were significantly associated with TC $(\beta=-1.454, P<0.033)$ and TGs $(\beta=-0.017, P<0.001)$, and $\operatorname{rs} 17123721$ was significantly associated with systolic $(\beta=1.701$, $P<0.010)$ and diastolic blood pressure $(\beta=0.984, P<0.022)$ after adjusting for covariates. Cox proportional hazard regression models showed that rs112077997 (HR: 0.90; 95\% CI: 0.82, 1.00) and rs 10789117 (HR: $0.90 ; 95 \%$ CI: $0.82,1.00$ ) were significantly associated with a lower risk of T2D after adjusting for covariates, whereas no association was observed for rs17123721.

Conclusions: Our results provide evidence for an association between genetic polymorphisms of ANGPTL3 and lower risk of type 2 diabetes, possibly through lifelong reduced concentrations of TGs and TC.

\section{Funding Sources}

This study was provided with bioresources from the National Biobank of Korea, the Centers for Disease Control and Prevention, Republic of Korea (KBP-2016-062). This research was supported by the Basic Science Research Program through the National Research Foundation of Korea (NRF), which is funded by the Ministry of Education, Science, and Technology (NRF-2015R1A2A1A15054758).

A Multivitamin/Multimineral Supplement Improves Indexes of Nutrition Status and Cardiovascular Health and Elicits Transcriptional Responses in Peripheral Blood Mononuclear Cells (P17-021)

Mark A Levy, Toni McKinnon, Howard Goldfine, Addison Enomoto, and John Cuomo

USANA Health Sciences

Background: There is considerable debate surrounding the health benefit(s) of dietary supplements, particularly multivitamin/ multimineral (MVM) supplements.

Objective: The objective of this study was to determine the effect of MVM supplementation on indexes of nutrition and antioxidant status and cardiovascular health, and to determine the effect of MVM supplementation on genome-wide gene expression in healthy adults. 
Methods: Twenty-four subjects ( $n=10$ female) were enrolled in a double-blind, placebo-controlled study for $4 \mathrm{wk}$. Fasting blood samples were collected prior to and at the end of the study.

Results: Indexes of nutrition status, including vitamins A, B6, B-12, D, E and K, were significantly increased following MVM supplementation. Additionally, 2 markers of cardiovascular health, HDL cholesterol and homocysteine, were significantly increased and decreased, respectively, whereas superoxide dismutase activity was significantly increased in MVM-supplemented individuals. Furthermore, we identified 97 genes ( $\mathrm{p}$

Conclusions: These data suggest that MVM supplementation can significantly improve numerous indexes of human health. Moreover, MVM supplementation may modulate gene expression patterns in peripheral blood mononuclear cells in healthy humans, which may reflect gene expression patterns in other organs/ tissues.

\section{Funding Sources}

USANA Health Sciences.

Genetic Variation of Lean Body Mass and Weight Loss in Response to Diet Interventions: The Preventing Overweight Using Novel Dietary Strategies Trial (POUNDS Lost) (P17-022)

Xiang Li, ${ }^{1}$ Dianjianyi Sun, ${ }^{1}$ Tao Zhou, ${ }^{1}$ Yoriko Heianza, ${ }^{1}$ George A Bray, ${ }^{2}$ Frank Sacks, ${ }^{3}$ and Lu Qi ${ }^{2}$

${ }^{1}$ Tulane University School of Public Health and Tropical Medicine, LA; ${ }^{2}$ Pennington Biomedical Research Center, Louisiana State University; and ${ }^{3}$ Harvard TH Chan School of Public Health, MA

Background: A recent genome-wide association study identified genetic variants that determine lean body mass; however, little has been clarified as to whether these genetic variants affect weight loss in response to dietary interventions.

Objective: We examined whether a genetic risk score (GRS) determining lean body mass was associated with diet-induced weight loss and changes in appetite measures.

Method: This study included 692 overweight and obese adults who were randomly assigned to 1 of 4 diets varying in macronutrient intake in the Preventing Overweight Using Novel Dietary Strategies Trial (POUNDS Lost). A GRS was calculated based on 5 single-nucleotide polymorphisms (SNPs) associated with lean body mass. An overall appetite score was calculated including measures of cravings, fullness, prospective consumption, and hunger. We investigated relations between the GRS and 6-mo changes in body weight, waist circumference, and the appetite measures.

Results: We found that the dietary fat intake (low compared with high) significantly interacted with the lean body mass GRS on changes in body weight, waist circumference, and the appetite measures from baseline to $6 \mathrm{mo}(P$-interaction $=0.015,0.024$, and

Conclusions: Our findings suggest that genetic variation of lean body mass may differentially affect adiposity changes according to dietary fat intakes, probably through the varied genetic effect on appetite.

This trial was registered at clinicaltrials.gov as NCT00072995.

\section{Funding Sources}

The study was supported by grants from the National Heart, Lung, and Blood Institute (HL071981, HL034594, HL126024), the National Institute of Diabetes and Digestive and Kidney Diseases (DK115679, DK091718, DK100383, DK078616), the Boston Obesity Nutrition Research Center (DK46200), and the United States-Israel Binational Science Foundation (2011036). LQ was a recipient of an American Heart Association Scientist Development Award (0730094N).

Supporting Images/Graphs

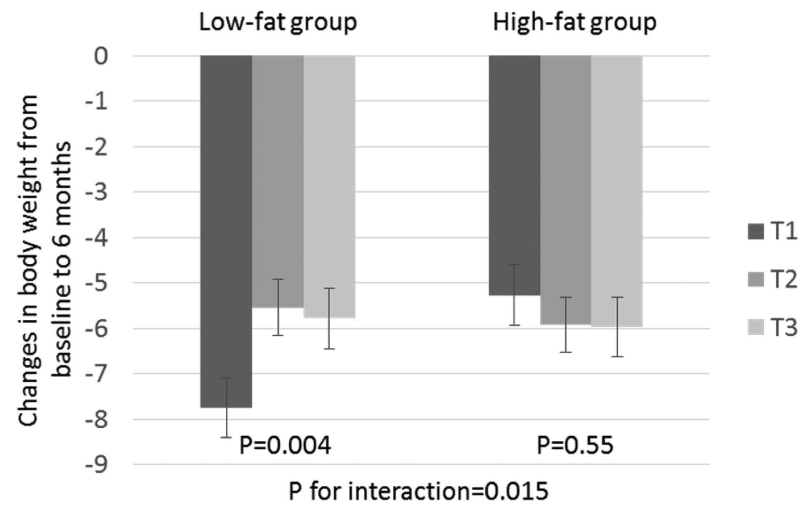

FIGURE P17-022-1 Effects of lean body mass GRS and high-/low-fat diets on changes in body weight at 6 months according to tertiles of lean body mass GRS. Values are mean \pm standard error after adjustment for age, race, sex, baseline BMI, and baseline energy intake. $T 1, n=203 ; T 2, n=28$; and $T r, n=$ 231.

The Correlation between Promoter CpG Methylation and the mRNA Levels of the Genes Regulated by Fatty Acids in Differentiated Adipocytes (P17-023)

\section{Yi Li, Elliott Cheng, and Md Masudul Alam}

Texas A\&M University-Kingsville

Adipose and muscle tissues are significantly affected in obesity and type 2 diabetes. Many of these changes are related to gene expression. In order to study the involvement of epigenetic modifications in mediating gene expression induced by hyperlipidemia, a condition occurring during the development of obesity and type 2 diabetes, 3T3L1 preadipocytes were induced to differentiate and cultured in media containing saturated fatty acids to mimic hyperlipidemic conditions during the differentiation process. DNA methylation sequencing was used to identify the methylated regions from the DNA samples isolated from the differentiated 3T3-L1 adipocytes treated with saturated and polyunsaturated fatty acids. In combination with genes previously identified as having their mRNA levels regulated by fatty acids, we have found an inverse correlation between DNA CpG methylation of the promoter region and the mRNA levels of a number of genes, including RASA2, MEF2C, and FAD3. We are currently individually validating the methylation status of these genes and also studying the signaling pathways mediating methylation of these genes.

\section{Funding Sources}

This research is supported by grant SC3GM116737 from the NIGMS of the NIH to YL. 
Imbalance in Maternal Dietary Ratio of Folate and Vitamin B-12 during Pregnancy Influences Expression of Imprinted IGF2/H19lncRNA via Epigenetic regulation (P17-024)

Aatish Mahajan, Divika Sapehia, and Jyotdeep Kaur

Postgraduate Institute of Medical Education and Research, Chandigarh, India

Objectives: To date there have been no studies to check the effect of altered dietary ratio of folate and vitamin B-12 during pregnancy on fetal growth. The present study was therefore designed to investigate the influence of dietary ratio of both these vitamins on the following: 1) the expression and promoter methylation of imprinted genes (IGF2/H19lncRNA) and associated miRNA; and 2) folate, B-12, and homocysteine concentrations, expression of folate transporters, and global DNA methylation.

Methods: Four-week-old C57BL/6 female mice (F0) were randomized into 9 diet groups (NBNF, NBFO, NBFD, BDNF, BDFD, BDFO, $\mathrm{BONF}, \mathrm{BOFO}$, and BOFD, where $\mathrm{NB}=$ normal $\mathrm{B}-12$; $\mathrm{NF}=$ normal folate; $\mathrm{FD}=$ folate deficient; $\mathrm{FO}=$ folate oversupplementation; $\mathrm{BD}=$ $\mathrm{B}-12$ deficient; and $\mathrm{BO}=\mathrm{B}-12$ oversupplementation) and were fed these diets for $6 \mathrm{wk}$; the males were maintained on normal diet. Mating was carried out and both male and female offspring (F1) fed the same diets were mated again and on day 20 of gestation maternal and fetal tissues (F2) were isolated. Folate, homocysteine, and B-12 concentrations were analyzed in serum. The expression of miRNA 675 and imprinted genes along with their promoter DNA methylation was studied. Expression of folate transporters RFC, PCFT, FOLR1, and global DNA methylation were studied.

Results: Circulating folate and B-12 concentrations were reduced, whereas homocysteine concentrations were high in F1 females of deficient groups $(P<0.01)$. Abnormal growth in fetuses was observed in both deficient conditions along with altered male/female ratios. In maternal and fetal tissues, overexpression of H19 was observed in deficient conditions along with decreased promoter methylation $(P<0.01)$. Biparental expression of $\mathrm{H} 19$ in F2 fetuses was found in NBFD and BDFD groups in spite of being transcribed from the maternal allele. In contrast, loss of IGF2 was observed along with increased methylation in both the F1 and F2 generations $(P<0.05)$ in deficient groups. miRNA 675 associated with IGF2/H19 locus was upregulated in maternal tissues under deficient conditions.

Expression of folate transporters, i.e., PCFT, RFC, and FOLR1 was increased in deficient groups $(P<0.01)$ along with a decrease in global methylation in maternal tissues except placenta $(P<0.05)$

Conclusion: Maternal dietary ratio of folate and vitamin B-12 during pregnancy influences fetal growth by altering the expression and promoter methylation of imprinted IGF2/H19lncRNA.

\section{Funding Sources}

Department of Biotechnology (BT/PR10671/PFN/20/811/2013), Government of India, PGIMER, Chandigarh.

\section{Supporting Images/Graphs}
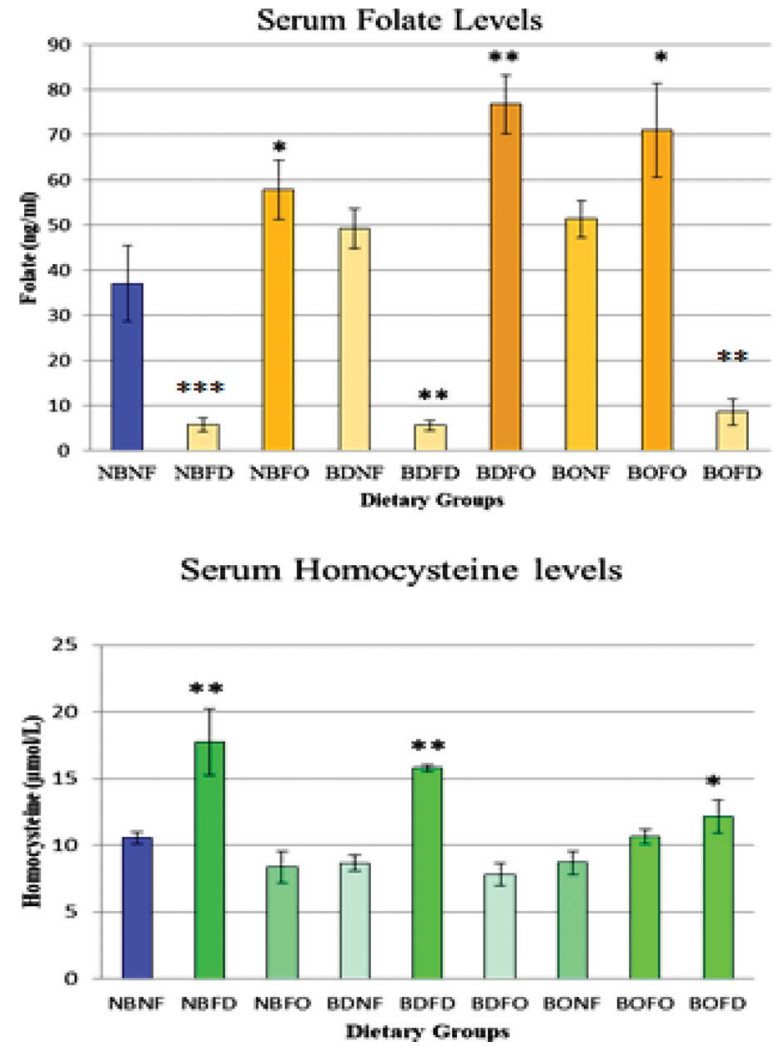

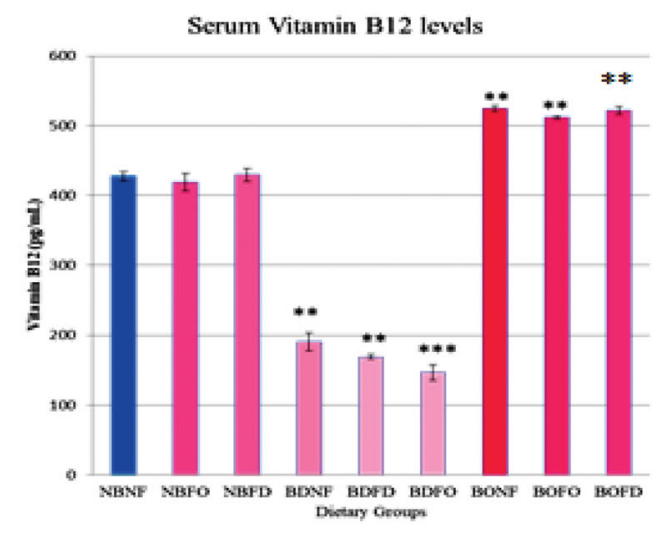

FIGURE P17-024-1 Circulating folate, B12 and Homocysteine levels in serum of F1 generation. $\left({ }^{\star} P<0.05,{ }^{\star \star} P<0.01,{ }^{\star \star \star} P<0.001\right.$ vs NBNF) 


\begin{tabular}{|c|c|c|c|}
\hline Groups & $\begin{array}{l}\text { No. } \\
\text { animals }=\mathrm{n}\end{array}$ & $\begin{array}{l}\text { Mean body } \\
\text { weight in } \\
\text { grams } \pm \mathrm{SD} \\
\text { (Initial) }\end{array}$ & $\begin{array}{l}\text { Mean body } \\
\text { weight in } \\
\text { grams } \pm \mathrm{SD}( \\
\text { AtMating) }\end{array}$ \\
\hline \multirow[t]{2}{*}{ NBNF } & Male: 4 & $22 \pm 1.6$ & $40 \pm 1.2$ \\
\hline & Female: 12 & $21 \pm 1.4$ & $36 \pm 1.8$ \\
\hline \multirow[t]{2}{*}{ NBFO } & Male: 4 & $23 \pm 1.9$ & $43 \pm 2.7$ \\
\hline & Female: 12 & $24 \pm 1.0$ & $37 \pm 1.8$ \\
\hline \multirow[t]{2}{*}{ NBFD } & Male: 4 & $23 \pm 2.7$ & $35 \pm 2.4$ \\
\hline & Female: 12 & $21 \pm 1.2$ & $33 \pm 1.4$ \\
\hline \multirow[t]{2}{*}{ BDNF } & Male: 4 & $22 \pm 1.1$ & $31 \pm 1.8^{*}$ \\
\hline & Female: 12 & $20 \pm 0.8$ & $30 \pm 1.6^{*}$ \\
\hline \multirow[t]{2}{*}{ BDFD } & Male: 4 & $21 \pm 1.7$ & $30 \pm 1.2^{*}$ \\
\hline & Female: 12 & $19 \pm 1.2$ & $31 \pm 1.4^{*}$ \\
\hline \multirow[t]{2}{*}{ BDFO } & Male: 4 & $20 \pm 1.4$ & $\begin{array}{l}27 \pm \quad 1.1^{* *} \\
\text { (Mostly died) }\end{array}$ \\
\hline & Female: 12 & $19 \pm 1.3$ & $\begin{array}{l}23 \pm \quad 1.3^{\star *} \\
\text { (Mostly died) }\end{array}$ \\
\hline \multirow[t]{2}{*}{ BONF } & Male: 4 & $23 \pm 2.1$ & $40 \pm 1.9$ \\
\hline & Female: 12 & $22 \pm 1.8$ & $40 \pm 2.1$ \\
\hline \multirow[t]{2}{*}{ BOFO } & Male: 4 & $24 \pm 1.6$ & $42 \pm 1.6$ \\
\hline & Female: 12 & $23 \pm 2.1$ & $40 \pm 1.7$ \\
\hline \multirow[t]{2}{*}{ BOFD } & Male: 4 & $22 \pm 1.9$ & $38 \pm 2.1$ \\
\hline & Female: 12 & $21 \pm 1.7$ & $37 \pm 1.9$ \\
\hline
\end{tabular}

\begin{tabular}{|l|l|l|l|}
\hline Group & $\begin{array}{l}\text { No. of } \\
\text { females } \\
\text { pregnant }\end{array}$ & $\begin{array}{l}\text { No. of } \\
\text { fetus/female }\end{array}$ & $\begin{array}{l}\text { Ratio } \\
\text { (M:F) }\end{array}$ \\
\hline NBNF & 11 & 8 & $4 \mathrm{M} / 4 \mathrm{~F}$ \\
\hline NBFO & 7 & 9 & $3 \mathrm{M} / 6 \mathrm{~F}$ \\
\hline NBFD & 4 & 6 & $2 \mathrm{M} / 4 \mathrm{~F}$ \\
\hline BDNF & 5 & 5 & $\mathbf{4 M} / 1 \mathrm{~F}$ \\
\hline BDFD & 3 & 6 & $\mathbf{4 M} / 2 \mathrm{~F}$ \\
\hline BDFO & 0 & 0 & 0 \\
\hline BONF & 12 & 15 & $7 \mathrm{M} / 8 \mathrm{~F}$ \\
\hline BOFO & 12 & 15 & $7 \mathrm{M} / 8 \mathrm{~F}$ \\
\hline BOFD & 10 & 8 & $5 \mathrm{M} / 3 \mathrm{~F}$ \\
\hline
\end{tabular}

TABLE P17-024-1 Body weights of mice for F1 generation and ratio of male/female born to F1 generation (mother). ( ${ }^{\star} P<0.05,{ }^{\star *} P<0.01$, $\star \star \star P<0.001$ vs NBNF)

RNA-Sequencing Identifies Polyunsaturated Fatty AcidResponsive Genes in the Juvenile Rat Cerebellum (P17-025)

Aaron Mehus, Aaron Dickey, Timothy PL Smith, Kathleen M Yeater, and Matthew J Picklo

USDA-Agricultural Research Service

Objective: RNA-sequencing (RNA-seq) is a powerful tool used to identify genome-wide differential gene expression in cells and tissues. Dietary n-3 ( $\omega-3)$ polyunsaturated fatty acids (PUFAs) influence postnatal brain growth and development; however, scant data exist regarding which genes are responsive to dietary n-3 PUFA depletion in the brain of growing animals. We tested the hypothesis that depleting dietary n-3 PUFAs would result in modifications to the cerebellar transcriptome of juvenile rats.

Methods: Three-week-old male rats ( $n=10$ /group) consumed ad libitum diets consisting of either $\alpha$-linolenic acid (ALA; 18:3n-3)-sufficient soybean oil (SO) or ALA-deficient corn oil (CO) for $4 \mathrm{wk}$. RNA-seq analysis was performed on the cerebellar tissue. Upper quartile (UQ) scaling and transcripts per kilobase million (TPM) data normalization methods, followed by respective ANOVA on normalized data, were evaluated and compared. To confirm the RNA-seq results, quantitative polymerase chain reaction (qPCR) was performed on the top 20 genes that were identified to be increased or decreased between the $\mathrm{SO}$ and $\mathrm{CO}$ groups. Cerebellar fatty acid (FA) content was analyzed.

Results: UQ scaling identified 100 genes and TPM normalization identified 107 genes that were differentially expressed between the two groups with $90 \%$ of the differences being $<2$-fold. Comparison of the two RNA-seq data normalization methods identified that 70 genes overlapped. Two genes (Nr4a3 and Smim17) that overlapped between the normalizations and one gene ( $G t f 2 f 2)$ exclusively identified by TPM were validated by qPCR. Nr4a3 was further validated in a separate cohort of animals. Cerebellar FA analysis demonstrated a 4-fold increase in n-6 docosapentaenoic acid (22:5n-6), an increase in arachidonic acid (20:4n-6), but no decrease in docosahexaenoic acid (22:6n-3) in the cerebellum of CO-fed animals.

Conclusions: This study identified several dietary n-3 PUFAresponsive genes within the postnatal cerebellum; however, only Nr4a3 could be validated in a separate cohort of animals. These data indicate that the cerebellar transcriptome of juvenile rats is tightly regulated with regards to dietary n-3 PUFA.

Funding Sources

This work was supported by USDA-Agricultural Research Service project 3062-51000-053-00D.

Effects of Fish Oil and Role of Uncoupling Protein 1 in Energy Metabolism for Diet-Induced Obese Female Mice (P17-026)

Emily K Miller, Mandana Pahlavani, Latha Ramalingam, Iurii

Koboziev, Shane Scoggin, and Naima Moustaid-Moussa

Texas Tech University

Objective: Brown adipose tissue (BAT) plays a critical role in energy expenditure through its thermogenic function. Therefore, its activation may help prevent or treat obesity. We have previously reported that eicosapentaenoic acid (EPA), an omega-3 (n-3) polyunsaturated fatty acid, significantly increased the thermogenic uncoupling protein 1 (UCP1) as well as gene expression of other key thermogenic markers in BAT of diet-induced obese male mice. However, whether female 
mice respond similarly to EPA has not been investigated. Further, it is unknown whether metabolic effects of EPA on BAT require UCP1 expression. We hypothesize that EPA reduces obesity and insulin resistance and activates BAT in female mice, and these effects are dependent on UCP1 expression.

Methods: We used wild-type (WT) and UCP1 knockout (KO) female mice housed at thermoneutral temperature $\left(30^{\circ} \mathrm{C}\right)$, a temperature shown to induce obesity in the $\mathrm{KO}$ animals. Mice were fed high-fat diets (HF) without or with EPA for up to 14 wk. Body weight (BW), body composition, and insulin tolerance tests were measured. Serum, BAT, and other tissues were harvested for future analyses.

Results: WT female mice (both HF and EPA groups) consumed significantly more food compared with the KO mice $(P<0.05)$. However, both WT groups gained significantly less weight than the two KO groups $(P<0.05)$. Additionally, in both WT and KO mice, EPA significantly reduced the total body fat percentage $(P<0.05)$ along with decreases in BAT and white adipose tissue (WAT) weights compared with HF-fed WT and KO mice. Furthermore, in both genotypes, insulin sensitivity was significantly higher in the EPA groups compared with the HF groups $(P<0.05)$.

Conclusions: These findings demonstrate that inactivation of the UCP1 gene in female mice dysregulates energy metabolism. Further, EPA protects female mice from diet-induced obesity, and these effects were independent of UCP1. Additional mechanistic studies in BAT and other tissues from these mice are necessary to determine mechanisms mediating the effects of EPA.

Funding Sources

NIH/NCCIH grant R15AT008879-01A1.

Coffee Consumption and Genetic Risk Score in Blood Pressure: Gene-Diet Interaction Analysis in a Population-Based Study (P17027)

Andreia M Miranda, Josiane Steluti, Marina Norde, Regina Fisberg, and Dirce Marchioni

School of Public Health, University of São Paulo, Brazil

Objective: This study aimed to investigate the interaction between usual coffee consumption and a genetic risk score related to high blood pressure, in a representative sample of São Paulo's population.

Methods: Data came from the Health Survey of Sao Paulo (ISACapital), a population-based, cross-sectional household health survey. The study sample included 533 participants aged $\geq 20$ y, living in São Paulo city, Brazil. Coffee consumption was assessed by two 24-h dietary recalls and categorized into $<1,1-3$, and $>3 \mathrm{cups} / \mathrm{d}$. The unweighted genetic risk score (GRS) was calculated based on 4 single-nucleotide polymorphisms (SNPs) [CYP1A1/CYP1A2 (rs2470893, rs2472297); CPLX3/ULK3 (rs6495122); MTHFR (rs17367504)] described in previous genome-wide association studies. Multiple logistic regression analyses were performed to estimate adjusted ORs and 95\% CIs for associations between high BP, and both high systolic blood pressure (SBP) and diastolic blood pressure (DBP) with the GRS. Thereafter, the effect of the interaction between the GRS and coffee consumption on high BP, SBP, and DBP was tested by including the respective multiplicative interaction term in the logistic regression models.
Results: All polymorphisms were in Hardy-Weinberg equilibrium. After adjustment for potential confounding factors, the findings suggested that higher GRS independently contributed to higher probability of elevated BP, SBP, and DBP in this population $(\mathrm{OR}=1.85 ; 95 \% \mathrm{CI}$ : $1.19,2.87$; OR $=2.30 ; 95 \% \mathrm{CI}: 1.32,4.01$; and $\mathrm{OR}=1.66$; $95 \% \mathrm{CI}$ : $1.10,2.51$, respectively). Moreover, there was a significant interaction effect for coffee consumption and GRS on high BP, and both SBP and DBP. A single-point rise in the GRS increases the probability of high $\mathrm{BP}(\mathrm{OR}=5.09 ; 95 \% \mathrm{CI}: 1.32,19.7)$ and both elevated SBP and DBP $(\mathrm{OR}=2.14$; 95\% CI: 1.12, 4.11; OR $=3.54$; 95\% CI: 1.17, 10.75), among participants with high coffee consumption ( $>3$ cups coffee/d).

Conclusions: Consumption of coffee could interact with genetic predisposition in relation to BP. Thus, individuals with greater GRS appeared to have high BP associated with higher coffee consumption, highlighting the particular importance of reducing coffee intake in individuals genetically predisposed to this cardiovascular disease risk factor.

\section{Funding Sources}

Financial support from São Paulo Research Foundation (FAPESP) (grant 2014/04540-2), and the from National Council of Technological and Scientific Development (CNPq) (procedurals 481176/2008-0, 473100/2009-6, and grant 306029/2011-1).

Does Paternal Exposure to Environmental Contaminants Affect the Metabolic Health of His Offspring and Future Generations? (P17-028)

Pauline Navarro, ${ }^{1}$ Nadine Leblanc, ${ }^{1}$ Bruno Marcotte, ${ }^{2}$ Janice Bailey, ${ }^{3}$ and Hélène Jacques ${ }^{1}$

${ }^{1}$ School of Nutrition and Institute of Nutrition and Functional Foods, ${ }^{2}$ Quebec Heart and Lung Institute, and ${ }^{3}$ Department of Animal Sciences, Faculty of Agriculture and Food Sciences, Laval University, Canada

Background: Chronic exposure to persistent organic pollutants (POPs) is associated with abnormal modifications in epigenetic regulation of glucose metabolism. This could induce the onset of insulin resistance and, thus, the development of type 2 diabetes, with repercussions for subsequent generations. Furthermore, as a methyl donor, folic acid (FA) could counteract the abnormal epigenetic modifications induced by toxic environmental compounds.

Objective: The objective of this study was to examine whether FA supplementation in pregnant rats exposed to POPs, at concentrations mimicking those observed in the Quebec Great Northern population, improves glucose metabolism in male rats of the first and the 2 subsequent generations via paternal transmission.

Methods: Sprague-Dawley female rats (F0) were exposed by gavage to either an environmentally relevant POP mixture or a control solution. Gavage continued during mating to unexposed males and pregnancy and stopped after birth of the first generation (F1). Four treatments were administered to F0 females: control + diet including $2 \mathrm{mg}$ FA $(n=10)$, control + diet including $6 \mathrm{mg}$ FA $(n=10)$, POPs + diet including $2 \mathrm{mg}$ FA $(n=10)$, POPs + diet including $6 \mathrm{mg}$ FA $(n=10)$. Prenatally treated F1 males were mated to untreated females to give birth to a second generation (F2) and then subsequently to a third generation (F3). After a 12-h fast, a glucose tolerance test was performed on F1, F2, and F3 
males at postnatal day (PND) 90 and 180. Plasma glucose, insulin, and C-peptide concentrations were measured at different time points $(T=0$, $15,30,60,90,120 \mathrm{~min})$.

Results: In F1 males at PND90 $(T=0)$, POPs increased plasma glucose. At PND180, a POP $\times$ FA interaction $(P=0.004)$ was observed on the incremental area under the curve (IAUC) molar insulin-to-Cpeptide ratio, showing that FA counteracted the low hepatic insulin extraction observed with POPs. In F2 males at PND90, a POP $\times$ FA interaction $(P=0.05)$ was also observed. Specifically, FA counteracted the increase of plasma IAUC insulin observed with POPs.

Conclusion: Prenatal exposure to POPs without FA supplementation may cause abnormalities in plasma glucose and insulin concentrations and hepatic insulin extraction that are transmitted from generation to generation by the paternal lineage. FA supplementation may counteract the deleterious effects of POPs.

\section{Funding Sources}

Supported by Canadian Institutes of Health Research.

\section{Multivitamin Supplementation Alters Peripheral Blood Mononuclear Cell Epigenetic Signatures Associated with Proinflammatory Cytokine Signaling (P17-029)}

Jessie Nicodemus Johnson

\section{USANA Health Sciences}

Background: A previous premarketing evaluation of USANA CellSentials supplementation in 70 individuals identified gender differences in response to supplementation.

Objective: The aim of this study was to characterize the cell-signaling pathways and investigate gender effects that are associated with supplementation in peripheral blood mononuclear cells.

Methods: A double-blind, randomized, placebo-controlled clinical trial (NCT03146312) was run at USANA Health Sciences in which healthy individuals were given placebo (11) or CellSentials (10) for 1 mo Genome-wide DNA methylation profiles from peripheral blood mononuclear cells were obtained with the use of an Infinium MethylationEpic BeadChip. Differentially methylated CpG sites were identified through the use of a paired mixed-effects linear regression model in the $\mathrm{R}$ package limma. Pathway analysis and immune cell enrichment were achieved with the use of Ingenuity Pathway Analysis and experimentally derived Functional element Overlap analysis of the ReGions from EWAS, respectively.

Results: A CellSentials-mediated epigenetic signature was observed among females but not males. Genes near CpG sites with reduced methylation after supplementation were enriched in a network centered on IL-1B and CD36 (network score $=27$ ) as well as enriched in monocyte DNase hypersensitivity sites $\left(P=4.48 \times 10^{-5}\right)$. Finally, the CellSentials-mediated epigenetic signature was enriched for associations with imprinted genes (empirical P-5), and these imprinted genes are enriched in a pathway centered on insulin response (network score $=55$ ).

Conclusions: The previous findings of gender differences in response to CellSentials supplementation were replicated; we also extended the observed gender differences to include the immune system, and highlighted a potential CellSentials-mediated immunomodulatory mechanism in females. Additionally, these results suggest that supplementation may be able to influence imprinted pathways associated, suggesting cell types and pathways for future study.

Funding Sources

USANA Health Sciences.

Effects of Butyrate on Histone H3 Acetylation around Disaccharidase Genes in the Rat Jejunum (P17-030)

Miyu Suzuki, Sayaka Suzuki, Saeko Takami, Kazue Honma, and

Toshinao Goda

University of Shizuoka, Japan

Objective: Both starvation and long-term intravenous nutrition reduce the function of the small intestine by causing a reduction in villus height and in the weight of gastrointestinal mucosa along with reduced expression of digestion- and absorption-related genes. Butyrate is known to inhibit histone deacetylases, and it is likely that butyrate promotes histone acetylation of the genes related to digestion/absorption and induces gene expression in the small intestine. In this study, we have investigated the effects of refeeding a diet containing sodium butyrate after starvation on the induction of disaccharidase genes in the jejunum of rats.

Method: Six-week-old SD male rats were fasted for $3 \mathrm{~d}$ and refed a high-sucrose diet (control) or a high-sucrose diet containing $5 \%$ sodium butyrate for 12 or $24 \mathrm{~h}$. The mRNA expression levels of disaccharidase genes were measured by real-time reverse transcriptase-polymerase chain reaction, and the amount of histone acetylation around the sucrase-isomaltase $(\mathrm{Si})$ gene and the lactase-phlorizin hydrolase $(\mathrm{Lph})$ gene, as well as the binding of acetylated histone binding protein BRD4, transcription elongation factor CDK9, and Cyclin T1 were measured by chromatin immunoprecipitation.

Results: Starvation for $3 \mathrm{~d}$ significantly decreased the histone $\mathrm{H} 4$ acetylation levels, as well as the binding levels of BRD4 and CDK9 around the $S i$ and $L p h$ genes. Refeeding a diet containing butyrate for $12 \mathrm{~h}$ led to an increase in the mRNA expression levels of $S i$ and $L p h$. Refeeding a diet containing butyrate for $24 \mathrm{~h}$ significantly increased the binding of BRD4 and CDK9 around the Si and Lph genes compared with the control.

Conclusions: Refeeding a diet containing butyrate enhances the expression of $S i$ and $L p h$ genes, although it increases the acetylation of histone $\mathrm{H} 3$ and the binding levels of the transcription elongation factor around the $S i$ and Lph genes.

The Influence of TAS1R1 Umami Taste Receptor Gene Polymorphisms on Dietary Consumption of Female Students at Mississippi State University (P17-031)

Pradtana Tapanee, Nicole Reeder, Ahmed Ch Saddam, Kristen Ensor, and Terezie Tolar Mosby

Mississippi State University

Objectives: Obesity is one of the main public health issues facing the state of Mississippi, where about one-third of young adults are obese. The factors that contribute to obesity are both genetic (40\%) and environmental (60\%). The purpose of this study is to investigate the influence of umami taste receptor gene polymorphisms from TAS1R1 
(rs17492553) on the dietary consumption of female college students in Mississippi.

Methods: A convenience sample of 203 female students from Mississippi State University with a mean \pm SD age of $20.71 \pm 3.12$ y participated in the study. An NIH Diet History Questionnaire was used to obtain dietary consumption data. Saliva samples were obtained and a TaqMan Sample-to-SNP Kit was used to analyze DNA extracted from saliva for single-nucleotide polymorphisms (SNPs). A Tanita body composition scale was used to estimate the percentage of body fat.

Results: Seventy-two women (35.47\%) had an elevated percentage body fat ( $\geq 30 \%)$. In this group, having the CC allele in the rs17492553 was significantly associated with an increased energy intake from carbohydrate $(P=0.018$, ANOVA) and with significantly lower energy intake from monounsaturated fat $(P=0.035$, ANOVA) compared with both the CT and TT allele groups. Women with a body fat percentage $\leq 30 \%$ ( $n=131,64.53 \%)$ had a significantly different intake of vitamin $\mathrm{C}$, niacin, and vitamin B-6 ( $P=0.047,0.03$, and 0.047 respectively, ANOVA), with subjects carrying the heterozygote having the highest intakes. There was no significant association between rs17492553 and other dietary intake. However, 2-way ANOVA revealed that there was a relationship between SNP variation and percentage of body fat on the value of protein intake by gram per body weight $(P=0.006)$ and on energy intake by kilocalorie per body weight $(P=0.017)$.

Conclusions: SNPs may contribute to the variation of dietary intake. These findings may be useful for developing personalized dietary recommendations for individuals in the future based on their individual genotype/phenotype to optimize health and prevent or treat obesity.

Evaluation of Telomere Length in Women with Severe Obesity Submitted to Roux-en-Y Gastric Bypass (P17-032)

Caroline R Welendorf, Carolina Nicoletti, Vitor C Pinhanelli, Marcela AS Pinhel, Leticia S Wolf, Flavia Ferreira, Rayana EC Foglietti, Bruno AP Oliveira, Wilson S Junior, and Carla B Nonino

University of São Paulo, Brazil

Objectives: Changes in nutrient absorption in patients undergoing Roux-en-Y gastric bypass (RYGB) may influence the telomere length (TL). In this context, the main objective of this study was to evaluate the TL in individuals with severe obesity before and 6 mo after RYGB.

Methods: We selected 18 women with severe obesity, aged 27$50 \mathrm{y}$, preparing to undergo RYGB. Anthropometric data (weight and height), body composition, and peripheral blood for analysis of TL were collected before and 6 mo after the surgical procedure. The TL was measured by a previously described polymerase chain reaction method, and the T/S ratio for each sample was obtained $[\Delta \mathrm{Ct}=\mathrm{Ct}(\mathrm{Tel})-$ Ct $(36 \mathrm{~B} 4)$; $\mathrm{T} / \mathrm{S}$ ratio $=2(-\Delta \mathrm{Ct})]$. For statistical analysis, the ShapiroWilk test, paired t test, and Pearson's correlation were used. Statistical significance was set for $P<0.05$. SPSS software was used to perform the analyses.

Results: A significant difference was observed in body composition in the preoperative period [fat-free (FFM): $64.7 \pm 7.5 \mathrm{~kg}$; FFM (percentage): $53.9 \% \pm 6.4 \%$; fat mass (FM): $52.6 \pm 10.8 \mathrm{~kg}$ and $\mathrm{FM}$ (percentage): $46.3 \% \pm 5.6 \%$ ] compared with the 6 -mo postoperative period (FFM: $53.8 \pm 7.2 \mathrm{~kg}$; FFM percentage: $61.9 \% \pm 7.5 \%$; FM: $33.8 \pm 9.7 \mathrm{~kg}$; FM percentage: $38.1 \% \pm 7.5 \% ; P<0.05)$. The TL was maintained 6 mo after surgery $(0.888 \pm 0.35)$, when compared with the preoperative period $(0.805 \pm 0.16 ; P>0.05)$.

Conclusions: The loss of weight beneficially changes anthropometric parameters and body composition. However, the amount of weight lost 6 mo after bariatric surgery does not significantly modify the TL.

\section{Funding Sources}

Conselho Nacional de Desenvolvimento Científico e Tecnológico (CNPq), Fundação de Apoio Ao Ensino, Pesquisa e Assistência do HCFMRP-USP (FAEPA).

Hypermethylation of Genes Involved in Appetite/Satiety Control after Roux-en-Y Gastric Bypass (P17-033)

Letícia S Wolf, Carolina Nicoletti, Vitor C Pinhanelli, Marcela AS Pinhel, Bruno AP Oliveira, Caroline Welendorf, Flavia C Ferreira, Rayana ED Foglietti, Wilson S Junior, and Carla B Nonino

University of São Paulo, Brazil

Objective: Knowledge of the DNA methylation pattern in the appetite/satiety pathway may promote understanding of the molecular mechanisms that determine the efficacy of any treatment for weight loss. Thus, the aim of this study was to evaluate changes in the pattern of methylation of genes related to the appetite/satiety pathway in women with severe obesity before and after Roux-en-Y Gastric Bypass (RYGB).

Methods: Ten women with severe obesity [body mass index $\left(\mathrm{kg} / \mathrm{m}^{2}\right)$ $>40$ ] submitted to RYGB were selected. Specific array-based DNA methylation analysis was performed with Infinium HumanMethylation $450 \mathrm{~K}$ Beadchip technology, which evaluated the methylation pattern of the genes related to the appetite/satiety pathway before and 6 mo after RYGB. The methylation level of each cytosine was expressed as a $\beta$ value (fluorescence intensity ratio of methylated to unmethylated alleles) ranging from 0 (unmethylated) to 1 (fully methylated).

Results: Hypermethylation of genes related to the appetite and satiety pathway was observed. Of these, the HCRTR1 (6.7\%), ADIPOR2 (5.1\%) and FTO (5.2\%) genes were the most hypermethylated $(P<0.05$, $P<0.001)$.

Conclusion: This study revealed that RYGB promotes significant changes in DNA methylation pattern, highlighting a hypermethylation of the HCRTR1, ADIPOR2 and FTO genes involved in the appetite/satiety pathway. This study will aid in the discovery of important biomarkers that are essential for clarifying mechanisms related to apetite/satiety in obesity.

Funding Sources

Fundação de Amparo à Pesquisa do Estado de São Paulo (FAPESP), Fundação de Apoio Ao Ensino, Pesquisa e Assistência (FAEPA).

Interaction between TCF7L2 SNP rs7903146 and Glycemic Load, Carbohydrate, and Dietary Fiber Intake in Relation to the Risk of Type 2 Diabetes in Cuban Americans (P17-034)

Ling Xu, Tan Li, Juan Liuzzi, Vijaya Narayanan, Gustavo G Zarini, and Fatma Huffman

Florida International University

Objectives: Type 2 diabetes (T2D) is a complex and chronic metabolic disorder that involves interaction between environmental factors and genetic predisposition. The TCF7L2 gene recently emerged 
as a top candidate gene for T2D. Studies have suggested the involvement of TCF7L2 in incretin hormone regulation, $\beta$-cell function, and hepatic glucose production. Differences in dietary factors, particularly carbohydrate intake influencing glycemic control, may modify some genetic association with T2D. Few studies have investigated such interactions in relation to the risk of $\mathrm{T} 2 \mathrm{D}$, and none have been performed on a Cuban American population. We hypothesized significant interaction between one common single-nucleotide polymorphism (SNP) rs7903146 $(\mathrm{C}>\mathrm{T})$ in the TCF7L2 gene and dietary factors in relation to the risk of $\mathrm{T} 2 \mathrm{D}$ and related phenotypes in Cuban Americans.

Methods: We genotyped TCF7L2 SNP rs7903146 from 341 Cuban Americans (172 without T2D/169 with T2D). Logistic regression was used to assess the association between SNP rs7903146 genotypes and the risk of T2D in a dominant model. Subgroup analyses were substratified by dietary factors (high compared with low). Interaction was tested by including the product of genotype $\times$ dietary factors in the model.

Results: We found a significant association between SNP rs7903146 and the risk of $\mathrm{T} 2 \mathrm{D}(\mathrm{OR}=1.65, P=0.031)$ in a dominant model. After including the interaction term in the model, a significant interaction was evident between dietary fiber intake and SNP rs7903146 polymorphism for the risk of T2D $(P=0.044)$. For subgroup analyses, SNP rs7903146 ( $\mathrm{OR}=2.43, P=0.010)$ significantly associated with the risk of T2D only for the subgroup with a higher dietary fiber intake (dietary fiber intake greater than or equal to the median), whereas no significant results were found for the subgroup with a lower dietary fiber intake. Further analyses in the subgroup with higher dietary fiber intake (controls only) showed that risk allele carriers have a significant A1C level $(P=0.044)$ compared with noncarriers.

Conclusion: The common TCF7L2 SNP rs7903146 variant was associated with increased risk of T2D in Cuban Americans, and the risk increased in the subgroup with a higher dietary fiber intake. Dietary factors may modify the genetic association with the risk of T2D and therefore need to be taken into consideration when conducting genetic association studies within populations.

Funding Sources

NIH/MBRS/SCORE AC\#124401529/42.

Ginger Exosomes Suppress NLRP3 Inflammasome Activation and Inflammatory Responses (P17-035)

Jiujiu Yu and Xingyi Chen

University of Nebraska Lincoln

Objective: The aim of this study was to screen and identify dietary exosomes that inhibit the activation of NLRP3 inflammasome.

Methods: Exosomes from different plants were purified by ultracentrifugation and incubated with bone marrow-derived macrophages. Their effects on NLRP3 inflammasome were evaluated by measuring interleukin $1 \mathrm{~b}$ in the medium and caspase- 1 autocleavage in the cell lysate, which are the 2 main downstream events of NLRP3 activation. The dietary exosomes that exerted strong inhibitory effects on NLRP3 inflammasome were further characterized, and their effects on NLRP3 inflammasome-mediated inflammatory responses examined in animal models.

Results: We found that exosomes purified from ginger strongly inhibited activation of NLRP3 inflammasome. The ginger exosomes were $\sim 134 \mathrm{~nm}$ in diameter. The inhibitory effects of ginger exosomes on NLRP3 inflammasome activation required long incubation $(>12 \mathrm{~h})$, suggesting that the cargos inside of the vesicles mediated the inhibitory effects. Most importantly, the administration of ginger exosomes in mice ameliorated NLRP3 inflammasome-mediated inflammatory responses.

Conclusion: The study identified that ginger exosomes inhibited NLRP3 inflammasome activation in vitro and in vivo.

Funding Sources

NIH P20GM104320 Nebraska Center Prevention of Obesity Diseases (NPOD) seed grant and project leader grant. 